\title{
Explaining the face-inversion effect: the face-scheme incompatibility (FSI) model
}

\author{
Sam S. Rakover
}

Published online: 5 February 2013

(C) Psychonomic Society, Inc. 2013

\begin{abstract}
The face-inversion effect (FIE) can be viewed as being based on two kinds of findings. According to the face (UI) effect, perception and recognition are better for faces presented upright $(\mathrm{U})$ than for faces presented inverted (I). According to the face/object(UI) effect, inversion impairs the processing of faces more than the processing of nonfacial objects (e.g., buildings or cars). Part I of this article focuses on the face(UI) effect and the configural-processing hypothesis, which is considered the most popular explanatory hypothesis of the FIE. In this hypothesis, it is proposed that inversion impairs the processing of configural information (the spatial relations between features) but hardly (if at all) impairs the processing of featural information (e.g., eyes, nose, and mouth). Part II of the article starts from the conclusion reached in part I, that the configural-processing hypothesis has not succeeded in explaining a substantial number of the findings and in resolving certain theoretical problems. The part then goes on to outline a new alternative model, the face-scheme incompatibility (FSI) model, which contends with these theoretical problems, accounts for the configural-processing hypothesis, succeeds in explaining a considerable portion of the empirical findings related to the face(UI) effect, and proposes a relatively new research program on the concept of the face scheme. The basic assumption of the FSI model is that schemes and prototypes are involved in processing a visual stimulus of a face and in transforming it to a "meaning-bearing" face, and that different schemes are involved if the face is presented upright or inverted.
\end{abstract}

Keywords Face-inversion effect · Face perception · Face recognition $\cdot$ Face-scheme $\cdot$ Face-prototype

S. S. Rakover $(\bowtie)$

Department of Psychology, Haifa University, Haifa, Israel 31905

e-mail: rakover@psy.haifa.ac.il

\section{Introduction}

The face-inversion effect (FIE) can be viewed as being based on two kinds of findings:

(a) Comparison of upright and inverted faces Perception and recognition are better of faces presented upright (hair above and chin below) than of faces presented inverted (chin above and hair below). This finding is called here the "face(UI)" effect - that is, FU - FI > 0, where F stands for face, $U$ for upright position, and I for inverted position.

(b) Comparison of faces and objects Inversion impairs the perception and recognition of faces more than of nonfacial objects (e.g., trees, buildings, cars), a finding denoted here as the "face/object(UI)" effect - that is, (FU - FI) > (OU - OI), where a nonfacial object is denoted O.

(See Freire, Lee, \& Symons, 2000; McKone \& Robbins, 2011; Rakover, 2002; Rakover \& Cahlon, 2001; Rossion \& Gauthier, 2002; Tanaka \& Gordon, 2011; Valentine, 1988; and Yin, 1969. Some researchers have proposed that the FIE is dependent on memory processes; see Leder \& Bruce, 1998b; Valentine, 1988).

To illustrate the strength of the face(UI) effect, consider Fig. 1. As can be seen in the figure, even knowing who owns the upside-down face apparently does not facilitate its perception: contrary to the upright face, difficulties arise in perceiving the inverted face; that is, the perception of the inverted face is opaque, whereas the perception of the upright face is transparent.

Research on the FIE can roughly be divided into two central lines. One of them, concerned with the face(UI) effect, tries to assess the kind of facial information whose processing is impaired by inversion (see Bruyer, 2011). It centers on a debate between two popular hypotheses, the configural-processing hypothesis and the holistic hypothesis, on which I shall go into in detail later (e.g., Leder \& 


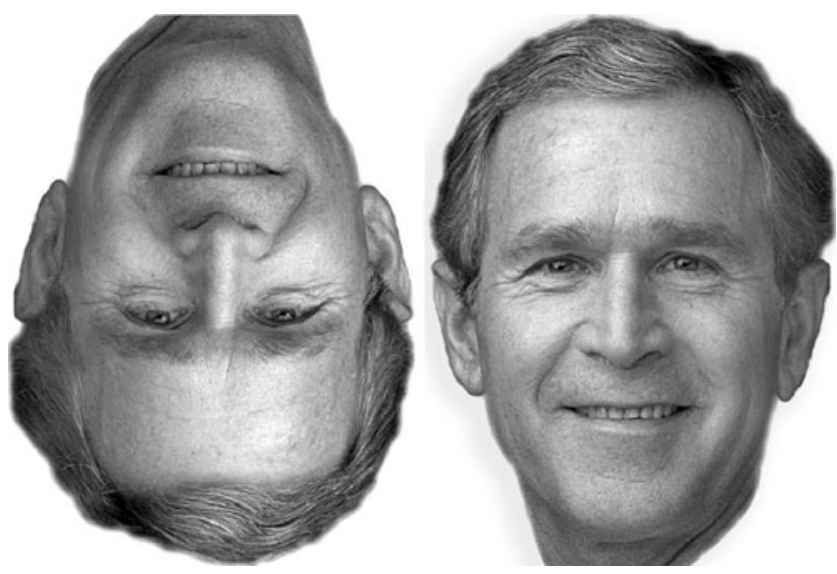

Fig. 1 The face of former United States President George W. Bush, presented both upright and inverted. Understanding that Bush's face is inverted does not facilitate its recognition

Bruce, 1998a, 1998b, 2000; Rossion, 2008, 2009; Searcy \& Bartlett, 1996). The other, which treats the face/object(UI) effect, investigates whether it is possible to perceive this finding as evidence that face processing occurs by means of a specific mechanism. Valentine (1988) wrote: "It is this finding, reported by Yin (1969), which has been cited as evidence that face recognition is a 'special' process" ( $p$. 472). This line of research centers on the debate between two other popular hypotheses, namely the face-specific mechanism hypothesis and the expertise hypothesis (e.g., Diamond \& Carey, 1986; Gauthier \& Bukach, 2007; Gauthier \& Tarr, 1997; McKone \& Robbins, 2007, 2011; Robbins \& McKone, 2007).

The intention in the present article is not to discuss research on the face/object(UI) effect, but instead research on the face(UI) effect alone. ${ }^{1}$ It focuses on the explanatory efficiency of this effect, discusses the above two hypotheses (the configural-processing and holistic hypotheses), and examines the former in particular. Given that research on the face(UI) effect has not succeeded in explaining all of the empirical findings and their associated theoretical problems, a relatively new theoretical model, the face-scheme incompatibility (FSI) model, is proposed. This model explains many relevant empirical findings and certain theoretical problems; it suggests interpretations for the configuralprocessing and holistic hypotheses, and also proposes a relatively new research program based on the concept of the face scheme.

\footnotetext{
${ }^{1}$ In addition to the fact that many studies have dealt only with the face (UI) effect (see Bruyer, 2011), it should be emphasized that the face/object(UI) effect raises a different research question. While the face (UI) effect is associated with the question of why (or how) inversion impairs the perception of faces, the face/object(UI) effect is associated with the question of why (or how) inversion impairs face perception and object perception differentially.
}

The article is divided into two parts. In Part I, I set out to check whether the face(UI) effect has been successfully explained. In Part II a new model is proposed, the FSI model, since the discussion in part I had shown the explanation of the face(UI) effect to be unsatisfactory both empirically and theoretically. ${ }^{2}$

Part I begins with the Types of Information and the Face (UI) Effect section, which briefly discusses the processing of different kinds of facial information. It focuses on the configural-processing and holistic hypotheses.

The Controversy Between the Configural-Processing and Holistic Hypotheses section discusses a series of studies that have attempted to decide between the two.

The Findings That Do Not Support the ConfiguralProcessing Hypothesis section discusses several studies that have not accorded with this hypothesis.

The Part I Discussion section considers whether progress has been made in understanding the face(UI) effect, and raises two major theoretical problems, mainly to do with the configural-processing hypothesis.

Part II starts from the position that research to date has failed to adduce a theory that explains all of the empirical findings or resolves the theoretical problems of this research. I then go on to outline the FSI model, which contends with these theoretical problems by envisioning face recognition as a subsystem of the general visual system. The model is demonstrated with several illustrations that substantiate it; this discussion focuses on the model's ability to explain empirical findings and the configural-processing hypothesis, and proposes a relatively new line of research based on the concept of the face scheme. The model's major assumption is that schemes and prototypes transform a visual stimulus of a face into a "meaning-bearing" face under two conditions: when a face is presented upright and when it is presented inverted.

\section{Part I: A critical review of the face(UI) effect}

Types of information and the face(UI) effect

The FIE literature, which primarily deals with the face(UI) effect, centers on two hypotheses: the configural-processing hypothesis and the holistic hypothesis (e.g., Bartlett, Searcy, \& Abdi, 2003; Diamond \& Carey, 1986; Loftus, Oberg, \& Dillon, 2004; Maurer, Le Grand, \& Mondloch, 2002; McKone, 2010; Rakover, 2002; Rakover \& Cahlon, 2001;

\footnotetext{
${ }^{2}$ The article is based on a set of laboratory studies with normal participants. Note that the FIE has been treated in a very large number of studies that have used it as a research tool serving diverse purposes, such as the investigation of cognitive changes in people who have suffered brain damage. I refer to these studies when they clearly touch on an explanation for the present phenomenon. Although a complete literature review is beyond the scope of the present study, an attempt will be made to review the most relevant experiments.
} 
Rossion, 2008, 2009; Tanaka \& Farah, 2003; Tanaka \& Gordon, 2011).

\section{Configural-processing hypothesis}

According to the configural-processing hypothesis (also called the dual-mode hypothesis), faces are perceived and remembered by means of two types of facial information: featural and configural. These undergo two different cognitive processes, in which greater importance is attributed to processing configural information than featural information. Featural information refers to the facial features: hair and forehead, eyes, nose, mouth, and chin. Configural information refers to the spatial relations of the facial features (e.g., the intereye distance or the distance between the nose and the mouth). Peterson and Rhodes (2003) defined configural information thus:

In the face perception literature, the term "configural" refers to spatial information. . . . It refers to conjoint information regarding components such as the eyes, nose, mouth and chin and the spatial relations between them. . . . The span of configural information can be small (e.g., specifying the relationship between two adjacent components) or it may be large (e.g., specifying the relationship between nonadjacent components separated by large distances, or specifying the relationship among all of the components in the face) (p. 4).

The configural-processing hypothesis is based on two main processes:

The effect of inversion on processing facial information Face inversion greatly impairs the processing of configural information and weakly impairs (if it impairs at all) the processing of featural information. A face presented upright is processed in terms of both of these types of information, while an upside-down face is processed mainly in terms of featural information. Therefore, perception and recognition are better of an upright face than of an upside-down face. Hence, two important predictions arise, which are supported by a number of studies:

(a) Changes in the facial configural information (e.g., changes in the distance between the eyes or between the nose and the mouth, or local inversion of the eyes and mouth, as in Thompson's illusion, 1980) are perceived/recognized better in the upright than in the inverted position. Furthermore, the disruption in processing facial information in the transition from the upright to the inverted position is not monotonic, because it is associated with the transition from processing configural and featural information to processing featural information alone. (b) Changes in featural information (e.g., darkening or coloring of the eyebrows and mouth) are perceived/recognized about equally when the face is presented either upright or inverted. That is, inversion has minor or no adverse effects on processing featural information (e.g., Bartlett \& Searcy, 1993; Ingvalson \& Wenger, 2005; Leder \& Bruce, 1998b, 2000; Leder \& Carbon, 2006; Murray, Rhodes, \& Schuchinsky, 2003; Murray, Yong, \& Rhodes, 2000; Rhodes, Brake, \& Atkinson, 1993; Searcy \& Bartlett, 1996).

More empirical findings that support the distinction between processing featural and configural information have shown the following: The blurring of the face and the mingling of features influence these two kinds of information differentially; featural information is processed before configural-holistic information; processing of configuralholistic information requires a greater investment of attention than does the processing of featural information; infants' sensitivity to featural information precedes their sensitivity to configural-holistic information; and children tend to rely on featural information more than on cognitiveholistic information (e.g., Carbon \& Leder, 2005; Collishaw \& Hole, 2000; Mondloch, Le Grand, \& Maurer, 2002; Palermo \& Rhodes, 2002; however, for critical discussions of the development issue, see Lee, Anzures, Quinn, Pascalis, \& Slater, 2011, and McKone, Crookes, \& Kanwisher, 2009).

Processing the information of faces and objects Faces are perceived and remembered by means of two types of information, featural and configural, and nonface objects by means of their parts or components. Due to this difference in the processing of visual information, one would expect the inversion of objects to exercise a lesser effect than does inversion of faces (e.g., Biederman, 1987; Biederman \& Kalocsai, 1998; Diamond \& Carey, 1986; Hummel, 2003; Leder \& Carbon, 2006; McKone, 2010; McKone, Martini, \& Nakayama, 2003; Tanaka \& Farah, 1993, 2003).

\section{Holistic hypothesis}

According to the holistic hypothesis, a face is perceived and remembered as a single whole, which is hard to break down into its parts (i.e., into featural and configural information). In their 1993 article, Tanaka and Farah wrote, "the representation of a face used in face recognition is not composed of representations of a face's parts, but more as a whole face" (p. 226).

Two interpretations of this approach may be suggested, one the "moderate holistic interpretation" and the other the "extreme holistic interpretation." The former holds that the parts and their spatial relations in a face are processed at a level below that of processing the whole face. For example, 
recognition of an isolated part of the face does not exceed recognition of this part when it appears in the face itself. According to the extreme interpretation, the whole face cannot be broken down into its components, which are not represented separately in the cognitive system (e.g., Leder \& Bruce, 1998a, 1998b, 2000; Searcy \& Bartlett, 1996; Tanaka \& Farah, 1993, 2003; Tanaka \& Gordon, 2011).

Like the foregoing hypothesis, the present one is based on two processes:

\section{The effect of inversion on processing facial information} Inverting the face greatly impairs the processing of holistic information. As compared with recognition of a nose that appears in the context of the whole face presented upright, recognition of a nose presented alone upright, inverted, or in a scrambled face is impaired (e.g., Tanaka \& Farah, 1993). In addition, configural changes in the eyes also impair recognition of the nose and the mouth when they appear in the context of the whole face upright, but not when the face is inverted (e.g., Tanaka \& Sengco, 1997), and face recognition is impaired when the face is learned in parts (i.e., the frame of the face and the eyes, nose, and mouth are presented separately) and not in its entirety (e.g., Farah, Tanaka, \& Drain, 1995; for a review, see Tanaka \& Gordon, 2011).

More findings that have supported holistic processing of the face are the following: A face composed of two parts of different faces (upper part from one face and lower part from another face) is perceived as a new face upright, but not inverted (see Fig. 8a below); a holistic process can be isolated in an inverted face by certain techniques that impair processing of the featural information, but not in an upright face; and high positive correlations have been found between the recognition of configurally/holistically altered faces and featurally altered faces when these are presented upright, but not when they are presented inverted (e.g., McKone et al., 2003; Young, Hellawell, \& Hay, 1987; Yovel \& Kanwisher, 2008).

Processing the information of faces and objects Faces are represented in the cognitive system in their entirety, while nonface objects are represented by means of their components. Hence, inversion will not affect objects as it affects faces, because objects are not represented in the cognitive system holistically (see, e.g., Tanaka \& Farah, 1993, 2003; Tanaka \& Sengco, 1997; but see Davidoff \& Donnelly, 1990; Donnelly \& Davidoff, 1999, for different findings and interpretations).

Farah (1992) and Tanaka and Farah $(1993,2003)$ suggested that visual forms may be placed on a holistic-tofeatural continuum, where recognition of faces necessitates a holistic representation, recognition of nonface objects necessitates a lesser-degree holistic representation (and a higherdegree representation by means of their components), and recognition of words does not necessitate a holistic representation, but rather representation through their components.

\section{Discussion}

By the nature of these two hypotheses, they offer different kinds of manipulations or tasks that participants are asked to perform in an experiment. According to the configuralprocessing hypothesis, it is standard to regard the effect of manipulation of the intereye or nose-mouth distance on face recognition as attesting the processing of configural information, and feature manipulations such as darkening eyebrows or pupils as attesting the processing of featural information. According to the holistic hypothesis, it is usual to regard the two following tasks as revealing expressions of the effect of holistic information on face recognition. In the part/whole task, recognition of a single facial feature (e.g., a nose) is better when the feature is shown in the context of the whole face rather than in isolation (e.g., Tanaka \& Farah, 1993). In the composite-face task, one is presented with a face whose upper part is from one person (e.g., former President Bush) and whose lower part is from another person (e.g., former President Clinton; see Fig. 8a below). Recognition of Bush's face in the case in which the two parts of the face are properly aligned is harder than in the case in which the bottom part (Clinton's) is not properly aligned with the upper part, but is slightly skewed, or in the case in which the face is presented inverted (see, e.g., Young et al., 1987).

Nevertheless, it is worth emphasizing that the different phenomena obtained by means of these manipulations or tasks should not be treated as the ultimate evidence for the two hypotheses. This approach is liable to create a situation in which testing (to support or refute) the configuralprocessing hypothesis, for example, will not be possible. If in a certain experiment we do not obtain the effect of inversion, it could be argued that the participants did not treat the stimuli presented to them as faces, but instead processed the stimuli as nonface objects.

Tanaka and Gordon (2011, note 1) referred to holistic processing as

the process by which the featural and configural information are integrated into a face representation. We use the term "configural processing" in a more limited sense, to refer to encoding of metric distances between features (i.e., second-order relational properties).

This definition of holistic processing raises a question about the relation between the perception of parts of the face and the holistic perception of the face. Several studies have tended to show that the holistic perception of a face is more than the total perceptions of its parts. For example, Rakover and Teucher (1997) found that prediction of face recognition 
in the upright position on the basis of recognition of five facial features separately (hair and forehead, eyes, nose, mouth, and chin) was lower than recognition of the face as a whole; Yovel, Paller, and Levy (2005) found that recognition of the face as a whole in the upright position was better than recognition of the right half or of the left half of the face separately. However, Gold, Mundy, and Tjan (2012), who used the "optimal Bayesian integrator" mathematical model, found that the perception of a face is no more than the sum of the perceptions of its isolated features. Note that although Penry (1971), the inventor of the Photofit, may be considered a researcher who supported face perception as being based on the sum of the perceptions of the parts, he suggested that a change in one of the facial features alters the perception of the entire face (Rakover \& Cahlon, 2001, called this phenomenon "Penry's effect"). So this suggestion appears more in line with the holistic hypothesis than with the perception of the face as the sum of the perceptions of its parts.

The controversy between the configural-processing and holistic hypotheses

As may be seen from the review so far, the configuralprocessing and holistic hypotheses can both offer fairly good explanations for the phenomena associated with the face(UI) effect. So what is the theoretical-empirical difference between them? An answer may be surmised by means of the following three lines of research: (1) a holistic or differential effect, (2) the effect of the facial context, and (3) "fusion" effects. Generally, the research findings have led to mixed conclusions.

\section{A holistic or differential effect}

According to the extreme holistic interpretation, the different kinds of facial information are represented in the cognitive system in a unified and indecomposable manner. Leder and Bruce (2000) wrote:

Thus the extreme holistic position is one where a face pattern is not decomposed into local features at all, and thus spatial relationships between these features will not be explicitly represented. (p. 515; see also Cabeza \& Kato, 2000)

So, by this interpretation we would expect that face inversion would impair all of the facial information, and differential impairments between configural and featural information would not be obtained. A similar prediction is suggested by Valentine's (1991) approach, namely that inversion causes a general disturbance in processing facial information, and no distinction will be obtained between the impairments of featural and configural information (see Searcy \& Bartlett, 1996). By contrast, according to the configural-processing hypothesis, we would expect inversion to impair configural, but not featural, information. As a whole, the research findings have supported the configuralprocessing hypothesis, but not the holistic hypothesis or Valentine's (1991) approach (e.g., Bartlett \& Searcy, 1993; Leder \& Bruce, 1998a, 1998b, 2000; Leder, Candrian, Huber, \& Bruce, 2001; Leder \& Carbon, 2006; Schwaninger \& Mast, 2005; Searcy \& Bartlett, 1996).

The question raised here is this: does the extreme holistic interpretation suit the research on the holistic hypothesis? My impression is that the moderate holistic interpretation is more suitable. (In a 2009 personal communication, Tanaka also wrote that the moderate interpretation is more suited to characterizing the holistic process.) First, Tanaka and Farah (1993) found that isolated parts of the face (e.g., a nose) could be identified, so it is hard to suggest that these parts are not represented in the cognitive system, even though identification of parts is better when they are presented within the whole face rather than in isolation. Second, Tanaka and Sengco (1997) found that a configural change in the eyes (the intereye distance) disturbed recognition of the nose and the mouth, a finding that attests to the dependence between featural and configural information in the representation of the whole face. This impression accords with what Farah, Wilson, Drain, and Tanaka (1998) wrote:

faces are represented holistically, which we define as meaning without explicitly representing (or relying to a lesser degree on explicit representations of) the local features themselves (p. 495).

On the assumption that the extreme holistic interpretation is inappropriate, the following question arises: Can the "differential effect" (that inversion impairs the processing of configural information much more than the processing of featural information) be predicted by the moderate holistic interpretation? One may propose a negative answer, because this interpretation does not determine explicitly that inversion causes the differential effect. If this interpretation did determine a differential effect, the configural-processing hypothesis would, in fact, be hardly distinguishable from the holistic hypothesis.

However, while the moderate holistic interpretation does not predict the differential effect, Rossion's $(2008,2009)$ hypothesis, the "perceptual field" hypothesis, does. Accordingly, inversion impairs holistic processing, which disrupts processing much more for the configural information than for the featural information. The holistic perception of an inverted face is constricted considerably, and as a result, processing the spatial relation between two features becomes much more difficult than processing a single 
feature. Hence, both the configural-processing hypothesis and Rossion's predict the differential effect.

The distinction between the configural-processing and holistic hypotheses has interesting implications for the relations among the types of facial information. The configuralprocessing hypothesis assumes a certain nondependence of the processing of featural and configural information, a distinction that is especially expressed in the inverted face position. But the holistic hypothesis suggests dependence between these two types of information, because of their very being together in the facial information, which functions as a single whole unit. Tanaka and Farah (2003) wrote:

In contrast to the relational view, the holistic view maintains that featural information and configural information are not distinguishable in the face representation. Hence, according to the holistic hypothesis, changes in one type of information (configuration) should affect memory for the other type of information (feature). (p. 63)

To support this empirical dependence, these researchers turned to the study of Tanaka and Sengco (1997), who found, as stated, that a change in the intereye distance influenced recognition of the nose and the mouth. By contrast, Maco and Leder (1988) presented empirical findings that supported the assumption of nondependence among the facial features, and Loftus, Oberg, and Dillon (2004) assumed, in their explanation of the FIE, nondependence of the processing of featural and configural information.

\section{The facial context effect}

According to the holistic hypothesis, the facial setting exerts a beneficial effect on recognition of its components. By contrast, it may be suggested from the configuralprocessing hypothesis that nondependence exists between the parts of the face, so that good recognition of a local configural change (e.g., in the intereye distance) will likely to be obtained even without the facial setting. The findings of the study of Leder, Candrian, Huber, and Bruce (2001) support the latter hypothesis. These researchers checked the effect of inversion on changes in the intereye distance, when the eyes appeared either in isolation or together with the various components of the face (with the nose or with the nose and the mouth). The components of the facial context were found to exert no effect on the perception of the size of the intereye distance in the upright or inverted position, and that the inversion effect centered on the eyes themselves; that is, the effect was local. Leder and Bruce (2000) also found that recognition of the local configural change presented in an isolated facial feature was equal to recognition of the same configural change that appeared in a whole face, a finding that supports the configural-processing hypothesis. By contrast, Leder and Carbon (2004) found that recognition of the configural change in a whole face was better than recognition of the same change in an isolated facial feature, a finding that accords with the holistic hypothesis. Leder and Carbon (2005) then found that not only did the facial context not aid recognition, under certain conditions it impaired it: After learning of the isolated parts of a face, recognition of these parts was impaired when they appeared in the full face, but not when they appeared in isolation. It seems that this holistic interference stems from the facial context, which imposes itself on information processing.

"Fusion" effects

These effects are of three kinds: conjunction, prototype, and composite.

The conjunction effect is based on a two-stage recognition experiment. In the first, study stage, a series of faces is shown. In the second, test stage, four types of faces are shown: old faces, which had been presented in the first stage; new faces, which had not been presented in the first stage; conjunction faces, which are composed of two different parts of two different faces shown in the first stage, in which one part contains internal features (eyes, nose, and mouth) of face $a$, and the other part contains the external frame (hair and forehead, cheeks, and chin) of face $b$; and "feature faces," which are likewise constructed of two different parts, one of which contains old features presented in the first stage of the experiment, and the other of which contains new features not shown in the first stage. Recognition of the old faces was greater than recognition of the conjunction faces, which in turn was higher than recognition of the feature faces, which itself was slightly greater than recognition of the new faces. These findings were interpreted as supporting the hypothesis that featural and configural information are coded independently, and that configural information requires greater attention than featural information does (e.g., Reinitz, Morrissey, \& Demb, 1994). Further studies showed that inversion impairs the old face/conjunction face distinction, because these types of faces are distinguished not by the features that appear in the first stage of the experiment, but by the configuralholistic relations (e.g., Bartlett et al., 2003; McKone \& Peh, 2006).

Bartlett, Searcy, and Abdi (2003), who described several studies on this phenomenon, wrote:

the encoding is configural and is spatially extended across the whole of an upright face (i.e., not just across the internal face-region). We view such spatially extended configural processing as essentially holistic (p. 32).

They proposed a theory (based on the neural net, anchored to the conceptual framework of Tulving, 1983, the general abstract processing system) that includes two 
separate tracks of processing information. The configural-holistic track offers an explanation for the distinction between recognition of old faces and of conjunction faces, and the featural track contributes an explanation for the distinction between recognition of conjunction and new faces.

The prototype effect is obtained in experiments of the following kind (e.g., Solso \& McCarthy, 1981). In the learning stage, a series of faces are presented. Each face is composed of some of the facial features of a prototype face. In the test stage, the prototype face is included in a series of test faces. Although the prototype was not shown in the learning stage, the participants mark it as an old face (that was presented in the learning stage). Because the faces in the learning stage were built from some of the features of the prototype face, it may be suggested that featural information is retained in memory. Cabeza and Kato (2000) showed, in the learning stage, a series of faces out of which two kinds of prototype faces were composed: one based on featural information, and the other based on configural information. They found in the test stage that both types of face were recognized as being old faces when presented upright, but not when inverted. Inversion impaired recognition of the prototype face on the basis of configural information.

The composite effect is obtained in experiments of the following kind (e.g., Young et al., 1987). Participants are shown a series of composite faces; that is, each is formed of two parts of pictures of famous people-for example, the upper part from a picture of former President George W. Bush and lower part from a picture of former President Bill Clinton (see Fig. 8a). Participants found it harder to identify the upper part when the composite face was presented upright than when it was presented inverted. In the upright position, participants were influenced by configural-holistic processes, and they perceived the composite as a new face. But when the face was inverted, these processes were impaired, and participants were able to identify the components of the face. Similar findings have been obtained in experiments in which participants were asked to identify emotions in faces (Calder, Young, Keane, \& Dean, 2000) and to make gender distinctions (Baudouin \& Humphreys, 2006).

\section{Discussion}

These three lines of research can be associated with the following two interpretations of the hypotheses under consideration. The holistic hypothesis suggests an interactive/integrative connection between featural and configural information, and between a local configural change and the other spatial relations in the whole face. By contrast, the configural-processing hypothesis suggests nondependence between featural and configural types of information, and between a local configural change and the other spatial relations in the whole face.

From these standpoints, the holistic hypothesis will apparently find it difficult to explain findings concerning the differential effects of inversion on the processing of featural and configural information (however, see Rossion, 2008, 2009). By contrast, it will be hard for the configural-processing hypothesis to explain findings concerning the effect of inversion on the relation between a local change and the whole face and on processing of the face as an entire unit.

These conclusions raise the following possibility: It may be that different processes are used by the participant to accomplish different tasks and experimental manipulations. For example, in the task revealing the conjunction effect, the participant uses different processes that are required for processing featural, configural, and holistic information. By contrast, he or she primarily processes (a) configural information in experiments in which the intereye or the nose-mouth distance has been changed, and (b) featural information when features have been changed.

Findings that do not support the configural-processing hypothesis

Face inversion is a manipulation that physically changes nothing in the face itself, but for the observer, inversion changes the up/down face directions when it is done in depth, and the up/down and left/right face directions when it is done on a plane. Thus, the marked effect of this manipulation on perception/recognition calls for explanation by means of an appeal to the activity of cognitive and neurophysiological systems.

The configural-processing hypothesis suggests that the cognitive system treats the information of an inverted face differentially: It fails in processing the configural information and succeeds in handling the featural information. Although several experimental findings have supported this hypothesis, the following three research programs narrow its scope of application.

The effect of inversion is not uniform and not always obtained

The effect that is predicted by the configural-processing hypothesis is not uniform, but depends on several factors, such as the type of facial information, the type of response, and the task that the participant in the experiment has to perform-factors about whose effect the present hypothesis has little to say. For example, Mäntylä and Holm (2005) found that inversion impaired recognition based on explicit recall, which was linked by a particular facial association, but not recognition based on knowledge connected with a general feeling and impression. Rakover (2008) found that 
inversion harmed the evaluation of a face as being pretty or attractive, but did not impair evaluation of a face as being interesting.

Goffaux and Rossion (2007) found that inversion impaired vertical configural changes (of the height of the eyes and eyebrows) more than horizontal changes (of intereye distance) (and see Barton, Keenan, \& Bass, 2001). Goffaux and Rossion explained this finding by noting that the face is arranged according to the vertical axis: eyes above nose above mouth. Sekunova and Barton (2008), who replicated Goffaux and Rossion's finding, found that the inversion effect was greatly attenuated when the vertical change was to the eyes alone, and the eyebrows remained as they were. They explained these findings by noting that the change in eye-eyebrow distance was made on an especially salient facial feature. Barton, Keenan, and Bass (2001) showed that the inversion effect on the nose-mouth distance decreased as the time of presentation of the facial stimulus increased, but that this effect increased with increases in nose-mouth distance (see also Kemp, McManus, \& Pigott, 1990).

Although the FIE is a broad phenomenon, it is not obtained under all experimental conditions. Loftus, Oberg, and Dillon (2004) noted several cases in which the phenomenon was not obtained-for example, in participants up to age 10 and in learning faces shown part by part. Loftus et al. themselves reported experimental findings in which faces were shown at extremely short latencies, where the FIE was obtained in recognizable faces of celebrities but not in unfamiliar faces. Burton and Jenkins (2011) found that the FIE obtained in familiar faces much greater than that obtained in unfamiliar faces.

Findings running counter to the configural-processing hypothesis

Valentine and Bruce (1988) found that the decline in face recognition as a function of change in facial position from $0^{\circ}$ to $180^{\circ}$ at intervals of $45^{\circ}$ was monotonic and did not suggest a qualitative change in the processing of facial information with a move from featural and configural processing to featural processing alone. But Murray, Rhodes, and Schuchinsky (2003) replicated Valentine and Bruce's experiment with intervals of $30^{\circ}$ and found that the decline in face recognition as a function of change in face position was not monotonic and did support an interpretation suggesting a qualitative change in the processing of facial information.

Rakover and Cahlon (in press) found that the similarity between scrambled faces in the upright and inverted positions is explained more accurately by a mathematical model based on first-order relational properties than by one based on facial configural information.
Sekuler, Gaspar, Gold, and Bennett (2004) found that the difference between processing upright and inverted face information was not qualitative, but quantitative. Riesenhuber, Jarudi, Gilad, and Sinha (2004) and Yovel and Kanwisher (2004) found that face inversion affected featural and configural information similarly. However, Rossion (2008) criticized these three studies. He proposed, for example, that the changes in featural information in the study of Riesenhuber et al. also involved configural changes (see also Rossion, 2009). In a review article, McKone and Yovel (2009) suggested that inversion influenced processing featural information to a degree that at times reached the level of the inversion effect on processing configural information. Tanaka and Gordon (2011) also found that inversion disturbed the processing of both kinds of information, featural and configural (see, as well, Amishav \& Kimchi, 2010; Kimchi \& Amishav, 2010).

\section{A necessary and a sufficient condition}

The configural-processing hypothesis has been tested in light of the following two questions:

(a) Is processing configural information a necessary condition for the face(UI) effect? That is, can an effect similar to the face(UI) effect be obtained by inversion of an isolated facial feature, even though the spatial relations between the facial features are eliminated?

(b) Is processing configural information a sufficient condition for the face(UI) effect? That is, is it possible for inversion of a face not to impair processing configural information? For example, can a change in configural information be perceived or recognized to the same degree in the upright and the inverted positions?

The research findings below answer both of these questions negatively: The inversion effect is obtained in situations in which the configural-processing hypothesis would not expect it (i.e., processing configural information is not a necessary condition), and inversion does not always impair processing of configural information, although such an impairment would be expected by this hypothesis (i.e., processing configural information is not a sufficient condition).

Necessary condition According to the configural-processing hypothesis, an isolated facial feature will not show behavior similar to the face(UI) effect, since the configural information has been removed. Nonetheless, Rakover and Teucher (1997) found that some isolated features elicited an effect like the face(UI) effect. The researchers ran an experiment on the recognition of isolated facial features: hair-forehead, eyes, noses, mouths, and chins. For example, at the study stage, the group of participants was shown a series of different and isolated eyes, one pair of eyes at a time. At 
the test stage, the participants were shown the old eyes (presented earlier) randomly mixed with new eyes (shown now for the first time). The task was to decide for each pair of eyes presented separately if it was new or old. Similar experiments were conducted with each of the other features, in which one group of participants saw the features upright, and a second group saw them inverted. The results showed that recognition of two of the inverted features - hair-forehead and eyes - was less than their recognition upright. That is, these two facial features showed an effect similar to the face(UI) effect. Hence, it seems that the spatial relations between features are not a necessary condition for the face (UI) effect (see the critique in Bartlett et al., 2003, and a response in Rakover, 2011a).

One way to save the configural-processing hypothesis would be to assume that (a) an isolated facial feature is in itself a visual form made up of basic components (e.g., an eye is composed of eyebrow, eyelid, eyeball, pupil, etc.) and their spatial relations, and (b) inversion also impairs recognition of an isolated facial feature because it disrupts the processing of the spatial relations among the feature's basic components (i.e., inversion disrupts the configural information within the eyes). This proposal, which may be viewed as the "extended configural-processing" hypothesis, can handle Rakover and Teucher's (1997) findings by pointing out that for face processing, the spatial relations within a facial feature are no less important than those between features.

Rakover (2012) found empirical support for the extended configural-processing hypothesis in a same/different experimental procedure: Recognition of isolated eyes was better when they were upright rather than inverted; that is, recognition of isolated eyes showed behavior similar to the face (UI) effect. Furthermore, perception and recognition of eyes tilted downward and upward (a within configural information change) were better when the eyes were upright rather than inverted. Hence, the spatial relations between the face features are not a necessary condition for the face(UI) effect, as inversion also impairs the processing of spatial relations within isolated eyes.

Sufficient condition Rakover (1999) found that in certain cases, the inversion of Thatcherized and scrambled faces in which the configural relations were changed not only did not decrease the degree of strangeness of the faces, but it even increased it - a finding that does not accord with the configural-processing hypothesis (see also Bruyer, 2011).

Schwaninger, Ryf, and Hofer (2003) found that the geometrical horizontal-vertical $(\mathrm{H}-\mathrm{V})$ illusion in a face resists inversion. (The $H-V$ illusion refers to a visual form in the shape of a $\mathrm{T}$ in which the horizontal line is perceived as being shorter than the vertical line, although the two lines are of equal length.) They proposed that the spatial relation between the eyes and the nose generates an illusion similar to the $\mathrm{H}-\mathrm{V}$ illusion, and found that the perception of this illusion is preserved even when the face is inverted. However, they restricted this finding to perceptual tasks, and the face(UI) effect to recognition tasks.

Rakover (2011a) showed in a series of experiments that a certain change in the configural information is not affected by face inversion in perceptual and recognition tasks. This informational change was produced by an increase or decrease by $20 \%$ in the size of the entire face, except for the eye area (see Fig. 2). As a result, the configural information between the features changed: For example, the distance between the eyebrows and the hair in the right-hand face in Fig. 2 became greater. These changes created an illusion that I call the "eye illlusion": The eyes in the left-hand face in Fig. 2 are perceived as being bigger than the eyes in the right-hand face, even though the sizes of the eyes in the two faces are equal. (The eye illusion was based on the structure of the famous geometrical illusion of Ebbinghaus/Titchener, in which a central circle surrounded by large circles is perceived as being small, while the same central circle surrounded by small circles is perceived as being largesee, e.g., Coren \& Girgus, 1978.)

In an experiment on faces reflected the eye illusion, participants were shown a series of pairs of faces (like the pairs of faces shown in Fig. 2). For each pair, they were asked to choose the face with the larger eyes. The faces were shown in two positions, upright and inverted, and in two experimental procedures. In the perceptual procedure, the two faces were shown side by side (as in Fig. 2); in the recognition procedure, the two faces were presented in succession.

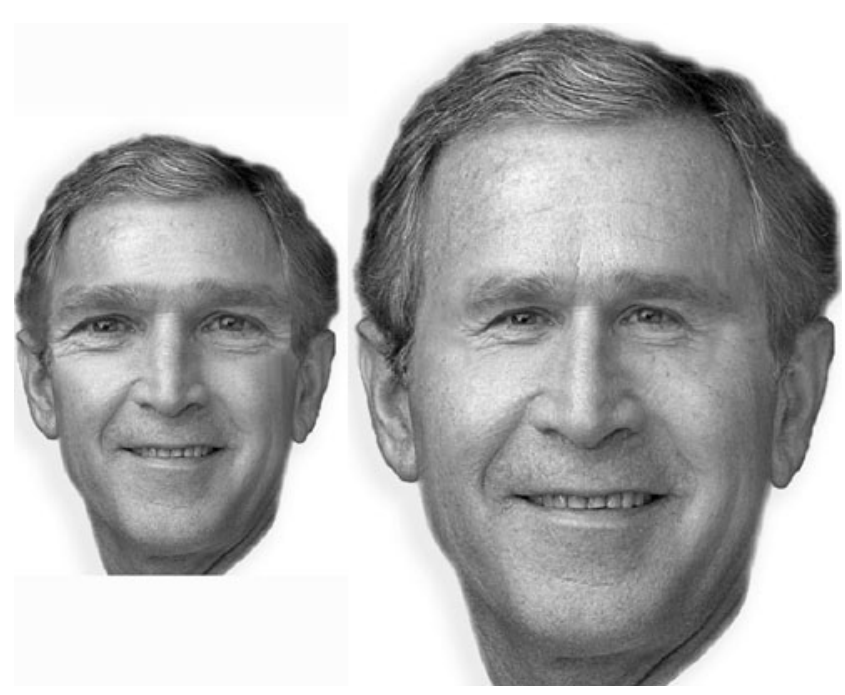

Fig. 2 The eye illusion, illustrated with the face of former United States President George W. Bush. The face has undergone changes in its configural information so that the eyes of the face on the left are perceived as being bigger than the eyes on the right, even though the sizes of the eyes in the two faces are identical 
The participants tended to choose in accordance with the illusion (e.g., regarding Fig. 2, they chose the lefthand face as having the bigger eyes), and upright and inverted positions of the faces did not differ in percentages of choice of the big eyes in either procedureperception or recognition. Hence, inversion in the present case did not eliminate the configural information, did not reduce the eye illusion, and did not cause an effect similar to the face(UI) effect.

\section{Part I discussion}

Besides the many studies that have supported the configural-processing hypothesis, several others have questioned it. This state of affairs may be treated in three different methodological ways (see Rakover, 1990).

(a) According to the strict hypothesis-testing methodology, the configural-processing hypothesis is disconfirmed: Some of the findings reviewed here did not accord with the predictions deriving from this hypothesis.

(b) According to the hypothesis-modification methodology, if the configural-processing hypothesis is not to be abandoned, because it is well entrenched both empirically and theoretically, one may proceed by means of the following two options: expand the hypothesis, as in the extended configural-processing hypothesis, or restrict the domain of the hypothesis's application, as in the possible proposal to exclude the configural information associated with visual geometrical illusions.

(c) According to the alternative-hypothesis methodology, an alternative hypothesis may be proposed that will provide explanations for most of the relevant findings and answer two important theoretical problems associated with the configural-processing hypothesis. The major purpose of part II is to present such an alternative model: the FSI model.

The FSI model is an attempt to handle the following two important theoretical questions. Both stress that the configural-processing hypothesis does not describe a cognitive process that will be needed to resolve these problems.

How does it come about that a visual stimulus is perceived as a face? The configural-processing hypothesis does not suggest a cognitive process that could explain how a given visual stimulus is perceived as a human face (and not as another form or object). The hypothesis just proposes that inversion impairs the processing of facial configural information, without offering an answer to this question. (The holistic hypothesis does not propose such a process, either-only that inversion impairs processing of the facial information as a whole.) ${ }^{3}$

How does it come about that especially the processing of configural information is impaired by inversion? The configural-processing hypothesis does not answer this question, either. It simply states that inversion harms mainly the processing of this kind of facial information, and not that of the featural information. (Note that the holistic hypothesis does not propose a similar differential effect caused by inversion. However, see Rossion, 2008, 2009.)

\section{Part II: Outline of the FSI model}

The FSI model is conceived here as representing a subsystem in the general system for processing visual information. A system is viewed as a general theoretical cognitive mechanism, within which various mechanisms that process visual information are organized (for discussions of particular models, mechanisms, and other related issues, see BailerJones, 2009; Bechtel, 2008; Rakover, 2007).

The FSI model is developed on the computational level (also called the functional level), and not on the algorithm or implementation levels (see Marr, 1982). The model is developed as an information-processing mechanism (which includes several submechanisms; see Fig. 3) in an attempt to provide answers to the two questions above and an explanation for the face(UI) effect. Fagan (2012) wrote, "It is widely recognized that there are no mechanisms simpliciter, but only mechanisms for some phenomenon $\mathrm{X}$. The phrase 'mechanisms for X' marks the target of explanation" (p. 457). The FSI mechanism, which is described verbally and diagrammatically, consists of different components that process visual information (faces and their parts). The components are organized and interact so as to produce the phenomenon under investigation. It is proposed that the description of the mechanism (the components and their activity) mirrors the actual activity that occurs in one's cognitive system when one processes facial information. While the mechanism's activity is automatic and beyond one's awareness, the phenomenon to be accounted forthe face(UI) effect-involves one's consciousness. (For a psychological explanations based on the mechanism, see

\footnotetext{
${ }^{3}$ The face-specific-mechanism hypothesis posits an innate special mechanism devoted to processing upright facial information, and the expertise hypothesis suggests a learning process to deal with such information. While both hypotheses can be potential sources for developing answers to our first question (how a visual stimulus is categorized as a face), it seems to me that neither of them proposes a description of the appropriate processes. Furthermore, both of these hypotheses may provide answers to the question of why inversion mainly impairs configural information processing, by anchoring this impairment to either a specific innate mechanism or a learning process.
} 
Fig. 3 The three processing stages of the FSI model. The thick-line rectangle in panel (a) represents the cognitive visual system in which all processes occur, including those in the wavy rectangle and in panel (b). The arrows in both panels (a) and (b) indicate the direction of information flow. (Other directions of information flow, which do not appear here, are considered in the Part II Discussion.) Thin-line rectangles include the relevant constructs and processes that produce the cognitive constructs that appear in the broken-line rectangles below them. In panel (b), the brokenline rectangle within the thinline rectangle contains the schemes and prototypes (generated in Stage 2) and their associated functions. These cognitive constructs and processes produce the upright and inverted faces that appear below them in the broken-line rectangle. (For details, see the text.) a Formation of Schemes and Prototypes

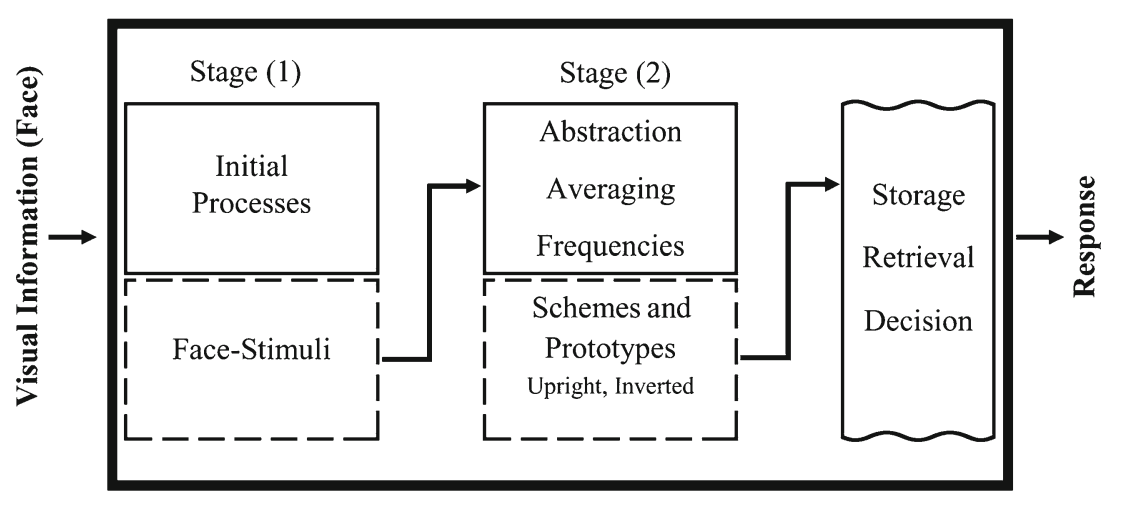

b Formation of a Meaning-Bearing Face

Stage (3)

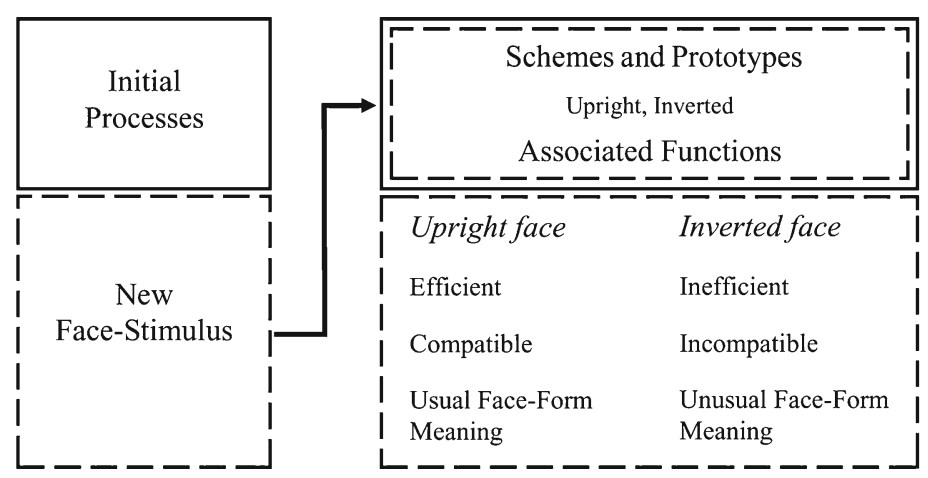

Bechtel, 2008; Bechtel \& Wright, 2009; Rakover, 2007, 2011b; C. D. Wright \& Bechtel, 2007). After its presentation below, the model will be substantiated with several illustrations, which open a relatively new line of research on facial schemes (see the Future Research section).

The model's development starts with an attempt to answer the first question above: How does one perceive a visual stimulus as a face? The cognitive processes, schemes, and prototypes suggested as an answer to this question explain the face(UI) effect, the configural-processing hypothesis, and also the second question above: How does inversion impair mainly configural information?

According to the FSI model, the visual information of a face undergoes three main transformation stages (see Fig. 3). All of the processes involved in handling the visual information of a face before the operation of schemes and prototypes (i.e., Stage 1) are called "initial" processes. Since these stages are conceived as being part of the cognitive system that handles visual information, several segments in its portrayal are based on well-known relevant information.

- Stage 1 Formation of the representation of a face stimulus from the visual stimulus by means of initial processes. (See Fig. 3a.)

- Stage 2 Formation of cognitive constructs of schemes and prototypes from the face stimuli by means of two cognitive processes: (a) abstraction and averaging, and (b) the frequencies of face stimuli appearing in upright and inverted positions. (See Fig. 3a.)

- Stage 3 Formation of a meaning-bearing face from a new face stimulus by means of schemes and prototypes (generated in Stage 2) and their associated functions: (a) comparison of a new face stimulus with the schemes and prototypes, (b) bestowal of face-form meaning on the new face stimulus by schemes and prototypes; and (c) interpretation and organization of the new face stimulus by schemes and prototypes. These cognitive constructs and functions are applied to a new face stimulus, which can appear in either the upright or the inverted position. (See Fig. 3b.)

These transformations are based on the following major proposal, which constitutes the FSI model's uniqueness: schemes and prototypes (already generated) are involved in processing a new face stimulus and transform it into a meaning-bearing face; this involvement differs in the cases of a face presented upright or inverted.

\section{Stage 1: Formation of a face stimulus}

Many initial processes join in handling the visual information of a face before the schemes and prototypes come into 
play. It is suggested that processing this information is automatic, without one's awareness. For example, the visual facial information undergoes several transformations, from conversion of the electromagnetic energy of light into neural electrochemical impulses in the retina, to processing performed in the visual cortex.

Additional processes operating on the visual information of a face relate to perceptual grouping (e.g., Palmer, 2003; Palmer, Brooks, \& Nelson, 2003). In the perceptual organization of a visual stimulus, several classic perceptualgrouping processes participate, such as proximity and similarity, as do a couple of new processes proposed by Palmer and colleagues: common region (i.e., elements within the same region are perceived as one group) and element connectedness (i.e., elements physically connected are perceived as one unit). [Note that Palmer and colleagues believed that grouping processes are likely to operate not only at the early stages of processing visual information (such as the level of retinal images), but also at later stages; that is, these processes are involved in different stages of information processing. The grouping processes themselves are not understood well and need explanation, but this matter lies beyond the present discussion.] It is proposed that these grouping processes are involved in the perceptual generation of facial features and their spatial relations, which are delimited by means of an outline of the face as the single, whole unit of a face stimulus. Given these processes, four important kinds of visual facial information can be discerned: featural information (facial features); firstorder relational properties (e.g., eyes above the nose, which is above the mouth; and also the organization of a feature itself-e.g., brows above pupils); configural information, or second-order relational properties (spatial relations between facial features and within a facial feature; i.e., spatial relations between the components that comprise a feature); and finally, holistic information of the whole-face stimulus. It seems that organization of the featural information precedes organization of the holistic/configural information; indeed, several of the studies reviewed above have shown, for example, that featural information is processed before holistic/configural information, and that infants are more sensitive to the former than to the latter (e.g., Carbon \& Leder, 2005; Collishaw \& Hole, 2000; Lee et al., 2011).

\section{Stage 2: Formation of schemes and prototypes}

Every individual is exposed to thousands of face stimuli, which create in the cognitive system frequency distributions of face stimuli and their parts. These kinds of facial information are processed in two ways, so as to create schemes and prototypes: (a) by abstraction and averaging, and (b) by frequencies of the face stimuli in upright and inverted position.
Abstraction and averaging

Facial information is processed by way of abstraction and averaging, which yield two important theoretical cognitive constructs: schemes and prototypes (i.e., norms). A facial scheme is conceived here as an abstract construct that portrays the general structure of face stimulus. By means of abstraction processes, schemes are constructed on the basis of the characteristics common to all face stimuli, such as the structure of the features themselves and the spatial relations among them (hair and forehead above the eyes, which are above the nose, which is above the mouth, which is above the chin; each feature is perceived as a subscheme: see Rumelhart, 1984). Each scheme (or subscheme) is associated with a criterion of "applicability," which treats a variety of face stimuli. Accordingly, a face stimulus is encompassed by a scheme if certain parts of the face stimulus and their spatial organization suit the scheme (see also the discussion in the Future Research section). This criterion imparts flexibility to a scheme by allowing it to handle a diversity of facial information. For example, an inverted eye is perceived as being upright and strange, since the spatial organization of its components still suits the subscheme of the eye (e.g., the pupil still stays within the eye frame [eyelids]). (The criterion of applicability is used at Stage 3 for handling a new face stimulus.) Since schemes are created on the basis of groups of different size, one may order them in terms of their generality. For example, the face scheme for a human being is more general than the face scheme of being Chinese. And because a scheme is abstract and general, it is relatively stable and not easily subject to changes.

By contrast, a prototype is not abstract, but represents a real image of a face, and is updated as a result of the individual's exposure to new faces (see, e.g., Rhodes \& Leopold, 2011). A prototype is a member of a group of different faces, which has the highest number of properties common to all other members of the group, and it can be characterized by the central tendency of a distribution - an average - of such properties as color, skin texture, structure of the facial features, and so forth. The similarity of each member of the group to the prototype can be determined by a similarity mechanism, which calculates the number of components that are common to each member and the prototype (e.g., Rakover \& Cahlon, 1989, 2008; Tversky, 1997). This mechanism is also used to handle a new face stimulus (see the next section). After the face stimulus has undergone treatment by a scheme, the similarity mechanism determines the similarity between the face stimulus and the appropriate prototype, and thereby classifies the face stimulus as belonging to a certain group and as having particular facial properties.

Hence, it may be suggested that while a scheme is an abstract construct that portrays the facial properties 
characterizing a large group of faces, a prototype (norm) is a real image that represents the most common properties of a group of faces. ${ }^{4}$

\section{Frequencies of face stimuli}

It is reasonable to assume that the frequency distribution of face stimuli according to their position is not uniform: it is very large when faces are upright, and very small when they are inverted. Thus, the frequency of face stimuli decreases as a function of the size of angular change from upright $\left(0^{\circ}\right)$ to inverted $\left(180^{\circ}\right)$. Now, assuming further that processing efficiency (e.g., the speed of processing and the accuracy of discrimination between minute facial components) of the schemes and subschemes is connected to the frequency distribution of the face stimuli, it is reasonable to suggest the "efficiency assumption": A new face stimulus is treated first by the most efficient cognitive construct - that is, an "upright scheme (subscheme)," which is connected to the upright position - and only when this treatment is not successful does the facial stimulus undergo treatment by a cognitive construct of lesser efficiency-that is, an "inverted scheme (subscheme)," which is connected to the inverted position. That is, a new face stimulus and its parts are treated by upright and inverted schemes and subschemes, whereby those in the upright position are activated first, and those in the inverted position only when necessary (see the next section). ${ }^{5}$

It is also assumed that schemes and subschemes can be activated separately. However, a particular interesting relationship exists between a scheme and its subschemes (see also the Future Research section). Subschemes, which treat inverted eyes or mouth in a face stimulus, are influenced in

\footnotetext{
${ }^{4}$ A prototype is different from a same-face average, which is based on a group of faces of the same person in different situations. Burton and Jenkins (2011) suggested that averaging is the learning process responsible for the generation of a familiar face. Roughly, it may be suggested that schemes determine that a face stimulus is a face and bestow on it a face-form meaning; prototypes add to this unfamiliar face several properties as a member in a certain group; and the same-face average makes a face familiar (e.g., easy to identify).

${ }^{5}$ The present approach may be viewed as being based on a succession of situations between upright and inverted facial positions, in which the upright scheme is linked to one extreme and the inverted scheme to the other. However, since the main concern of the present study is to explain the difference in perception and recognition between upright and inverted faces, only these two positions are discussed. Note that one may consider different connections between face position, frequency of faces, and schemes. For example, one may propose that the efficiency of the upright scheme reduces as a function of a face's angular change (from $0^{\circ}$ to $180^{\circ}$ ). Nevertheless, I believe that the present proposal is better, since it allows for the generation of inverted schemes on the basis of the faces presented throughout life in the inverted position (as in the case in which one sees an inverted face of a person lying on her back from above).
}

the following way by the scheme that treats the rest of the face stimulus: The greater the processing efficiency of a scheme, the greater its ability to highlight the activity of the subschemes, hence the greater the saliency of the inverted eyes or mouth. (This relationship may be associated with the famous von Restorff effect; see, e.g., Fabiani \& Donchin, 1995. Accordingly, there is an increase in memory for an item that is out of its context.)

The difference in processing efficiency between upright and inverted face schemes can be illustrated by Fig. 1. As was stated above, even knowing who owns the upside-down face does not facilitate perceiving the inverted face as transparently as the upright face. The relationship between a scheme and a subscheme can be illustrated by Fig. 6 below. As is stated for that figure, the exchange of the human eyes and the gorilla's eyes makes their perception strange. The strangeness is more salient in the human than in the gorilla face, because one is exposed to human faces much more than to gorillas' faces. The same holds for locally inverted eyes: The weirdness of the eyes is greater in the human than in the gorilla face.

\section{Stage 3: Formation of a meaning-bearing face}

Given the creation of schemes and prototypes in Stage 2, how is a new face stimulus (which previously underwent Stage 1 processing) treated by these cognitive constructs in the upright and the inverted position? The answer is provided by a description of an intricate decision mechanism that employs the constructs of schemes and prototypes, as well as their associated functions: (a) comparison, (b) meaning bestowal, and (c) interpretation and organization. A brief summary of the decision mechanism is provided at the end of the portrayal of Stage 3 .

\section{Comparison}

If the new face stimulus accords with the face scheme (by meeting the criterion of applicability), it is processed as a face. After this, a prototype comes into play. That is, the face stimulus is treated first by a scheme, to decide whether this stimulus is a human face, and then further determinations are made about the face by means of its comparison with a prototype (through the operation of the similarity mechanism). The scheme is activated after being appropriately searched for among other schemes stored in the cognitive system; the search itself is initiated after the visual stimulus has been handled by the initial processes that generated the face stimulus (see Fig. 3a). With practice, this sequence of processing develops into an expert mechanism that reaches the highest level of performance - it is fast, automatic, and beyond one's awareness. 
One may suggest several ways to establish the kinds of comparisons required by the FSI model (i.e., the criterion of applicability and the similarity mechanism). The best known are those made by means of similarity and decision rules (e.g., Estes, 1994; Hintzman, 1986; Medin \& Schaffer, 1978; Navarro \& Lee, 2004; Nosofsky, 1984, 1992; Rakover \& Cahlon, 1989, 2008; Tversky, 1997). In this respect, the present approach (comparison of a new face stimulus with schemes and prototypes) pursues the idea that a given object is categorized by means of calculation of its similarity to a prototype (e.g., Rosch \& Mervis, 1975). Partial support for this proposal may be drawn from the following findings: Choices of upright or inverted scrambled faces as being similar to an upright normal face are predicted accurately by a mathematical model based on the first-order relational properties, which produces a similarity scale for the scrambled faces (see Rakover \& Cahlon, in press); the categorization process of a given visual stimulus as a face precedes identification of the face (e.g., Liu, Harris, \& Kanwisher, 2002); and typical faces are categorized as faces faster than distinctive faces (e.g., Valentine, 1991; see also Rakover \& Cahlon, 2001).

\section{Meaning bestowal}

As may be seen from Fig. 3b, the new face stimulus and its parts are compared with different schemes (whole face, eyes, nose, etc.) and prototypes. It is assumed that when a match is obtained (by using the criterion of applicability and the similarity mechanism), the schemes and prototypes confer on the new face stimulus "face-form meaning." That is, the face stimulus acquires the meaning of a face form (which is expressed in the conscious experience of perceiving a human face) by the schemes and prototypes: For example, one has the conscious experience that this visual stimulus is of a young blond woman with green eyes. (In most cases, the processes of comparison and meaning bestowal are executed very quickly, but in certain situations in which an ambiguous visual stimulus is presented, one may feel a strain to figure out what this stimulus means-i.e., to find the appropriate scheme for the ambiguous stimulus.)

Up to Stage 3-namely, the stage of comparison and meaning bestowal - the face stimulus has no meaning as a face. Just as under a microscope a living cell cannot be perceived as such until its structure has been properly learned (see Bechtel, 2008), a certain visual stimulus is imperceptible as a face until it has been compared with facial schemes and prototypes and is endowed with meaning. The visual stimulus of a face that appears before one's eyes is nothing other than a physical stimulus. In itself, it has no meaning. It acquires meaning only in one's mind. According to the FSI model, the scheme is the construct that confers upon this stimulus a face-form meaning (and the prototypes attribute additional meanings). This process of meaning bestowal rests on the function of the scheme of supplying understanding. On this topic, Rumelhart (1984) wrote, "Perhaps the central function of schemata is in the construction of an interpretation of an event, object or situation - that is, in the process of comprehension" ( $p$. 166). (See also Brewer \& Nakamura, 1984.) The process of the bestowal of meaning (which is expressed in a conscious experience of perceiving a human face) is very hard to describe and explain. McGinn (1991) stated that "the brain has some property which confers consciousness upon it; I do not say that I know which" (pp. 204-205). (For a discussion of this issue, see Rakover, 2007.) Nevertheless, it is suggested here that the bestowal of face-form meaning is a process associated with schemes and prototypes. ${ }^{6}$

\section{Interpretation and organization}

In most cases (as in the upright position), interpreting and organizing a new face stimulus are straightforward, in accordance with schemes and prototypes. However, in certain situations the new face stimulus does not match these cognitive constructs. Here I discuss two such situations, which are mainly related to the concept of scheme: (a) The whole face stimulus or some of its parts are inverted - a situation that is a main concern of the present article, and (b) a small number of parts in the face stimulus do not fit the scheme, even in the upright position. In both situations, an attempt is made to interpret and organize the incompatible parts in accordance with a scheme and subschemes.

Upright and inverted positions There are no incompatibilities between the schemes and the new facial stimulus when the stimulus is presented upright, but there are when the stimulus is presented inverted.

According to the efficiency assumption, because the upright scheme is the most efficient (since it is based on a huge number of upright face stimuli), the cognitive system uses it

\footnotetext{
$\overline{{ }^{6}}$ The suggestion that a scheme bestows on a new face stimulus a faceform meaning may be conceived of as being similar to the following approach in the philosophy of mind. It has been proposed that the consciousness of a mental state depends on a higher-order process involving awareness (see Kim, 2011). For example, Ruth is aware of her pain caused by a cut. If, however, her attention is powerfully attracted to another happening, she will discover the cut and the pain much later. Hence, a mental state becomes conscious when Ruth is aware of her own mental state - that is, when her mental state is perceived by a higher-order perceptual process, or when it is connected with Ruth's thought about this mental state. This approach has been criticized from many viewpoints. One important critique concerns the question of how to account for the consciousness of the higher-order process itself; an answer that is based on a higher higher-order process leads to infinite regress. I briefly suggest in the Part II Discussion (see below) a possible answer to a similar question that concerns the case of a scheme and a new face stimulus.
} 
first. The comparison between it and the new face stimulus presented upright is automatic, immediate, and easy; it does not create incompatibilities, and the face stimulus acquires a compatible face-form meaning-for example, this is a human face, these are the eyes, and so on. Similarly, Goldstein and Chance (1980) suggested that because the scheme develops as a result of exposure to a huge number of faces in the upright position, it will be hard pressed to deal with inverted faces, and because the individual is exposed to a huge number of faces of his or her own kind, a facial scheme develops in the cognitive system that is hard put to deal with other-race faces. After the comparison and bestowal of meaning, the facial information moves on to further processing stages (see the wavy rectangle in Fig. 3a). The information is stored in long-term memory and retrieved when needed.

Contrary to the situation for the upright position, in the inverted position, incompatibilities arise. According to the FSI model, the most efficient schemes - the upright schemes and subschemes - are used first for comparison and for conferring face-form meaning on the face stimulus, even in the case of inverted position. In this case, face-form meaning is conferred on the parts of the face stimulus that do meet the criterion of upright applicability. In an inverted face (e.g., the left of Fig. 1), these parts are the eyes and the mouth, since they show symmetry relative to the horizontal axis and retain an important part of their spatial organization in the inverted position. They are interpreted as facial features that are upright but odd. Rock (1973, p. 62) wrote about inverted eyes, "The eyes appear strangely shaped because their flatter part is on the top rather than the bottom, and they have dark markings (the eyebrows) underneath." However, the hair and forehead, the nose, and the face outline display no symmetry relative to the horizontal axis. Here, the upright subschemes find it hard to handle these inverted features, and the inverted subschemes are activated. Since their efficiency is low, they cannot process these features in the way that the upright schemes can, but they do foster the comprehension that these features are presented in the inverted position. Similarly, Rock (1973, p. 61) proposed that although the cognitive corrective mechanism is overtaxed when attempting to handle an inverted face, "the process of correction is successful insofar as it leads to identifying the face as a face."

The face-scheme incompatibilities increase in the case in which the eyes and the mouth appear inverted in an upright face stimulus. Here, the inverted eyes and mouth are treated by upright subschemes, and as a result these two features are perceived as being upright and strange (since they still meet the criteria of applicability). The rest of the upright face stimulus is handled by an upright scheme. As its processing efficiency is high, the two features become salient, and their strangeness is increased.
In contrast to the process above, the face-scheme incompatibilities decrease in the case in which the eyes and mouth appear upright in an inverted face stimulus. There, these two features match the appropriate upright subschemes, and the inverted scheme (which treats the rest of the face stimulus) has low processing efficiency.

Incompatible parts When a small number of parts in the face stimulus do not fit the scheme (while most parts do), an attempt is made to interpret, organize, and bestow meaning on these incompatible parts in terms of the scheme. That is, an attempt is made to subordinate the interpretation of the incompatible parts to the scheme. However, this attempt is not always successful (see Fig. 9 below for an example).

A brief summary of Stage 3-A decision mechanism of the FSI model

Given Stage 2, how are a new face stimulus and its parts (which previously underwent Stage 1) treated in the upright and inverted positions? In light of the discussion above, the answer is focused on schemes and subschemes and involves the following four situations:

- If a match between an upright new face stimulus and an upright scheme is achieved (by meeting the criterion of upright applicability), the face stimulus is perceived as an upright face (and is bestowed with face-form meaning).

- If in an upright face stimulus, the eyes and the mouth appear inverted (as in the Margaret Thatcher illusion), the stimulus is handled by an upright scheme and subschemes: The face stimulus is perceived as being an upright face, and the two features are perceived as being upright, but strange (because they still meet the criterion of upright applicability). Since an upright scheme's processing efficiency is high, it causes the two features to stand out and to increase their strangeness.

- If an inverted face stimulus appears, the eyes and mouth are handled by upright subschemes, and the rest of the face stimulus by an inverted scheme. Since the inverted scheme's processing efficiency is low, the perception of the eyes and mouth as upright and strange is not intensified, and the rest of the face stimulus is perceived as being inverted.

- If in an inverted face stimulus the eyes and mouth appear upright, these two features match the upright subschemes, and the rest of the face stimulus matches the inverted scheme.

Finally, the following brief comment must be made. When face from another race is presented, an own-race scheme is activated. However, since the own-race scheme 
is built on the basis of thousands of faces of one's own race, this scheme is not suited to handle a face of another race. Consequently, errors in perception and recognition are generated. In this respect, the present model is similar to the "contact" hypothesis, which suggests that the own-race bias can be accounted for by the degree of people's exposure to other-race faces - that is, the rarer the contact, the lower the recognition (e.g., Rakover \& Cahlon, 2001; Wright, Boyd, $\&$ Tredoux, 2003).

\section{Several illustrations}

The following five figures illustrate four important properties and implications of schemes and subschemes, as they are developed in this model: first, their power to organize and interpret faces and parts of faces; second, their ability to provide a new explanation for a known facial effect; third, their ability to easily interpret an inverted face and its parts, which can hardly be explained by the configural-processing hypothesis or by the assignment of top and bottom directions to a face and its parts; and fourth, their ability to suggest relatively new research in face perception and recognition (for a discussion, see the Future Research section below).

Figure 4 illustrates the organizational and interpretational power of a face scheme. In Fig. 4a, the face scheme creates the image of an angry man; in Fig. 4b, the image of a smiling man appears; and in Fig. 4c, which is composed of the two preceding figures, an angry man with a double chin appears. In the latter case, the face is so interpreted and organized that the smiling mouth in Fig. $4 \mathrm{~b}$ is perceived as being a double chin.

Figure 5 illustrates the organizational and interpretational power of a face scheme (and subschemes), as well as the explanatory weakness of the configural-processing hypothesis. In Fig. 5, which shows a cat-woman, we focus on the overall structure of the face, the hair, the eyes, the nose, and the mouth as components compatible with the upright scheme of a woman's face, and we interpret the cat's ears, nose, whiskers, and mouth in terms of this scheme as a
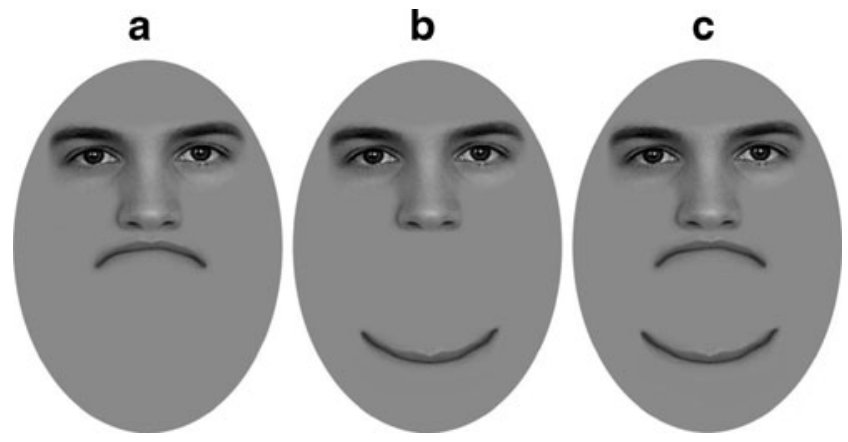

Fig. 4 Three egg faces (drawings by the author): (a) angry, (b) smiling, and (c) angry with a double chin necklace or a decoration on the woman's forehead. That is, the latter components are conceived in accordance with the configuration of a woman's face. Similarly, when the figure is inverted, we focus on the ears, eyes (despite the lines under the eyes), nose, whiskers, mouth, and hair around the head (fur) as components compatible with the upright scheme of a cat, and partially interpret the woman's nose and mouth on the cat's forehead as lines in the fur or as scars. In this case, the meanings of the latter components are subordinated to the cat configuration (but see Fig. 9). Note that since inversion impairs the processing of configural information, the configural-processing hypothesis would find it difficult to explain how it comes about that one perceives the inverted woman as a cat.

Figure 6 likewise illustrates the organizational and interpretational power of a face scheme (and subschemes) and the explanatory weakness of the configural-processing hypothesis. In Fig. $6 a$ and b, the human's and the gorilla's eyes have been exchanged, making their perceptions strange. This strangeness is more marked in the human than in the gorilla face, because the learning level of the scheme is far higher for the human face than for the gorilla face. For the same reason, eye inversion in the human face causes greater oddity than eye inversion in the gorilla's face (see Fig. 6c and d). These effects are difficult to explain in terms of the configural-processing hypothesis, mainly because this hypothesis does not deal with the relation between the degrees of exposure to a face (human vs. gorilla) and processing of its configural information.

Figure 7 illustrates the organizational and interpretational power of a face subscheme and the explanatory weakness of

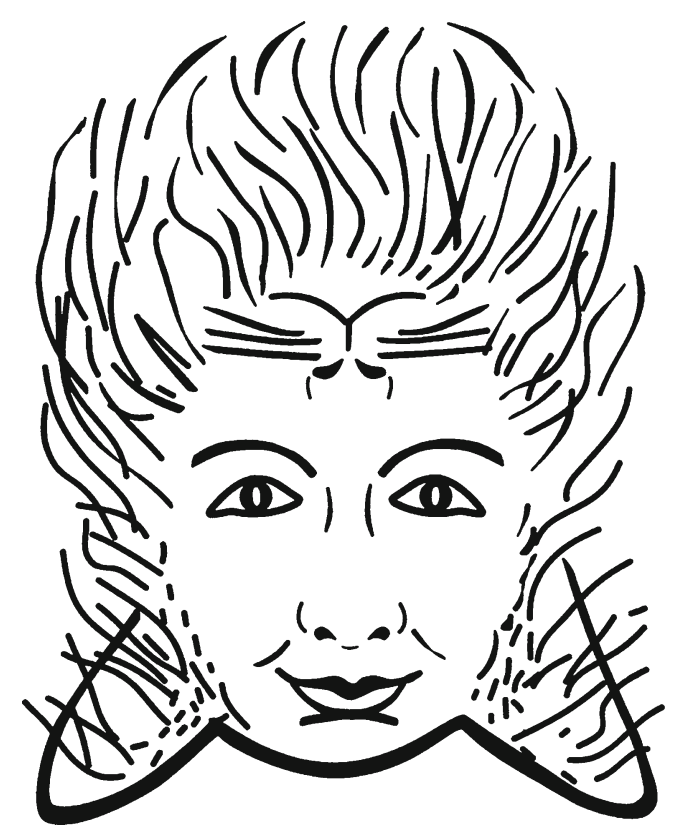

Fig. 5 Cat-woman (drawing by the author). Inversion of the woman's face shows a cat's face 

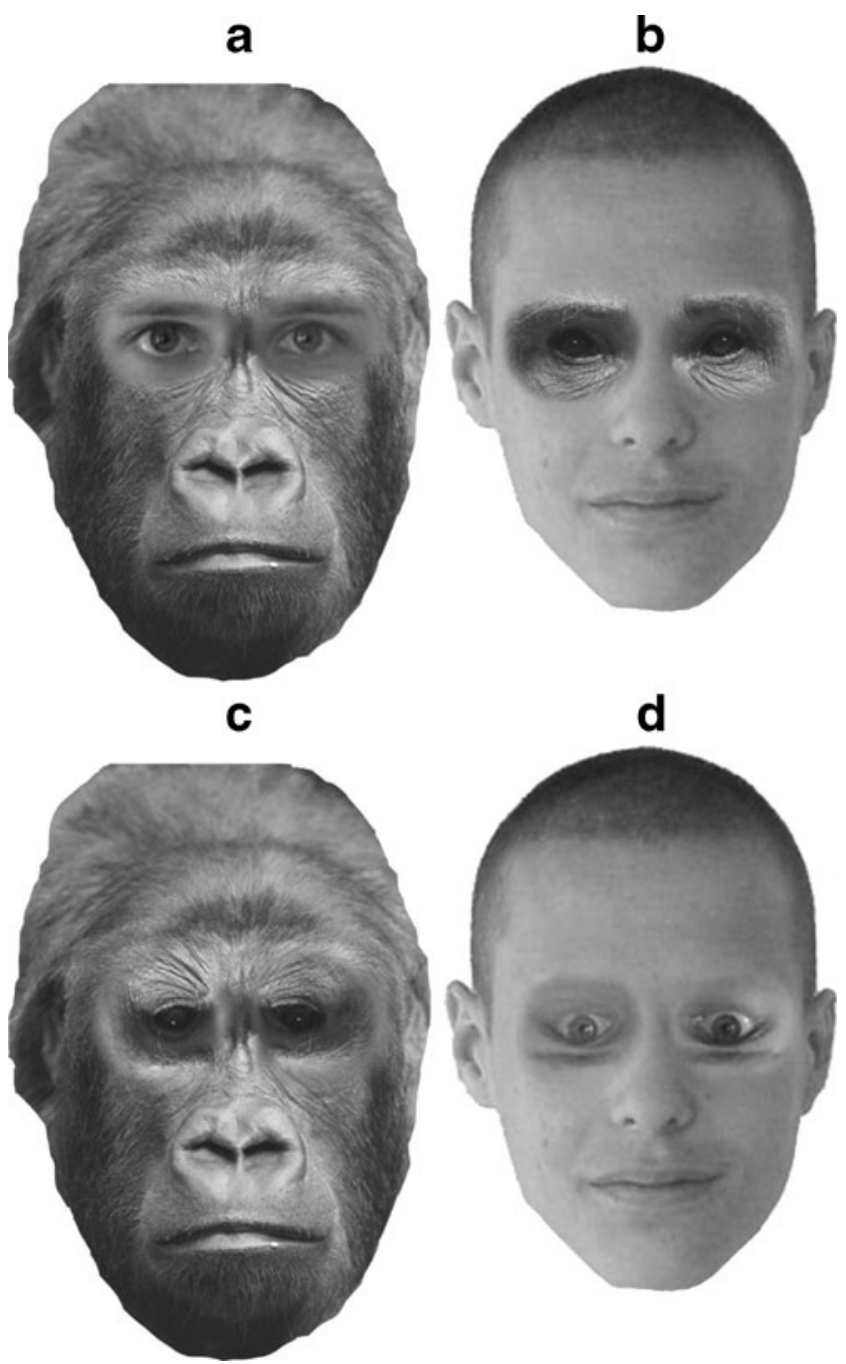

Fig. 6 Gorilla/man eyes. In pictures (a) and (b), the man's eyes are exchanged with the gorilla's eyes. The oddity of the man's face (b) is greater than that of the gorilla's face (a). Inversion of the eyes in the man's face (d) also elicits greater oddity than inversion of the eyes in the gorilla's face $(\mathbf{c})$

the configural-processing hypothesis. We interpret the visual stimulus in Fig. $7 \mathrm{a}$ as being composed of eyebrows, eyes, and bags under the eyes, in accordance with the subscheme of an upright eye, but when the eyes are inverted (Fig. 7b), in accordance with the subscheme of an upright eye, we interpret

a
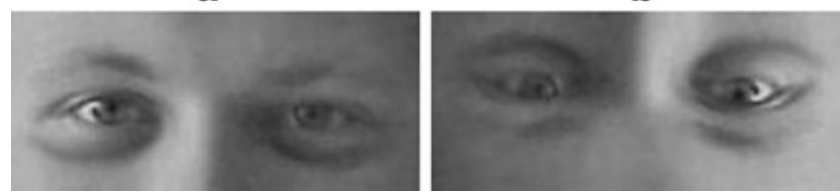

Fig. 7 Two different pairs of eyes. When the eyes are upright (a), the lines under the eyes are perceived/interpreted as bags under the eyes, but when inverted (b), the eyes are perceived/interpreted as being upright but strange; in the latter case, the lines under the eyes are perceived as eyebrows, and the eyebrows as bags under the eyes the bags under the eyes as eyebrows and the eyebrows as bags under the eyes. Similarly, Rakover (1999) suggested that the Margaret Thatcher illusion (Thompson, 1980) may be explained not by recourse to the configural-processing hypothesis, but by an appeal to the subscheme of the eye: We interpret inverted eyes as being strange eyes in the upright position. The decrease in strangeness with the inversion of the entire Thatcherized face stems from the eyes as a single perceptual unit returning to their regular, everyday position - a position compatible with the upright eye subscheme. Further support for the present interpretation may be obtained from an experiment by Parks, Coss, and Coss (1985), who found that inverted eyes and mouth aroused a sense of strangeness when those features were presented in isolation. Similarly, Rock (1988) noted that in an inverted face, the eyes appeared strange when the other parts of the face were covered.

Figure 8 illustrates how the present model may handle a known facial effect, as well as the explanatory weakness of the assignment of top and bottom directions to a face and its parts. The Thatcherized composites of presidents Bush (top halves of the faces) and Clinton (bottom halves of the faces) exemplify two interesting effects: the composite effect, and the Thatcher illusion discussed above (see Thompson, 1980; Young et al., 1987). As to the composite effect, Fig. 8a in the upright position illustrates the organization of the two facial parts into a perceptual whole. When the composite stimulus appears in the upright position, there is a perfect match between it and a highly efficient upright face scheme, which results in perception of the composite stimulus as an regular upright face. Consequently, it is difficult to discern the two facial parts of the known leaders. By contrast, when Fig. $8 \mathrm{a}$ is inverted, the matching is between it and an
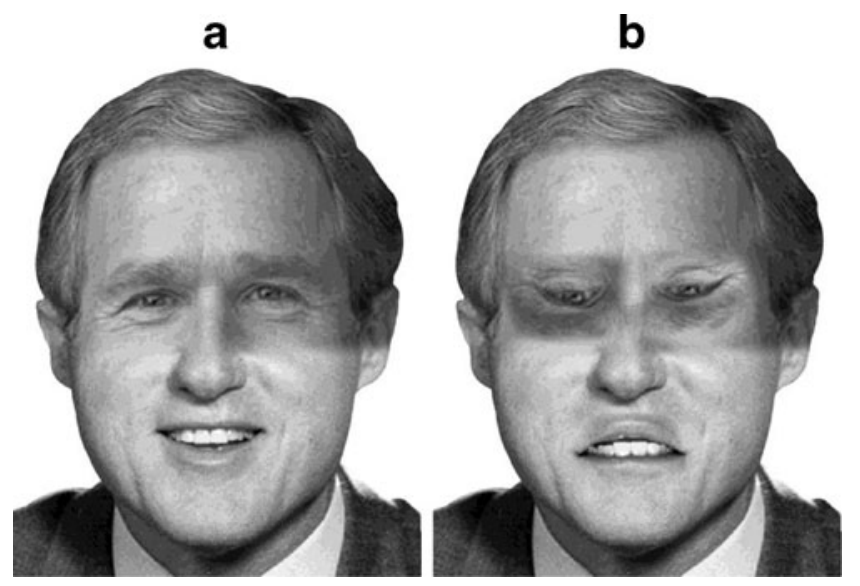

Fig. 8 Thatcherized Bush/Clinton composites. The upper part of each picture is of former United States President George W. Bush, and the low part is of former US President Bill Clinton. While in the upright position it is easier to identify the parts of the face in panel (a) than in panel (b), when the pictures are inverted it is easier to identify the parts of the face in panel (b) than in panel (a) 
inefficient inverted composite scheme and two efficient upright subschemes (eyes and mouth), which results in a perception of the stimulus as an inverted face with upright strange eyes and mouth. Hence, the holistic perception of the face is impaired, and the possibility of discerning the facial parts of the two known leaders increases. Given these effects, one may propose that while Young et al. suggested that the two halves of a composite "readily fuse to form an effective whole" and that "seeing a new configuration is sufficiently powerful that it can interfere with the identification of the individual features from which such composites are formed" (p. 748), the FSI model explains the "fusion" of the two halves into a "new configuration" in the following way: There is a complete match between an upright face scheme and the upright composite stimulus. Furthermore, while Young et al. accounted for the effect of inversion on the perception of a composite in terms of impairment of the configurational information, the FSI model proposes an explanation for this effect in terms of the match between the inverted composite stimulus, an inverted face scheme, and upright subschemes.

As for the Thatcher illusion, a comparison of the two faces in Fig. 8 shows that the parts of the faces of former presidents Bush and Clinton in Fig. 8a are easier to identify than those in Fig. $8 \mathrm{~b}$ because the eyes and mouth in $8 \mathrm{~b}$ are perceived as being upright and odd. But when the pictures are inverted, it is easier to identify the features in $8 \mathrm{~b}$ than in $8 \mathrm{a}$, because the features (eyes and mouth upright) in $8 \mathrm{~b}$ are more similar to the features of the two former presidents than they are in $8 \mathrm{a}$, in which these features are perceived as being upright and strange.

Figure 8 illustrates the difference between two explanatory approaches to face identification: the present approach and the approach that assigns top and bottom directions to a whole face and to its features (see Bartlett \& Searcy, 1993; Parks et al., 1985; Rock, 1973, 1974). First, let us examine the case in which the faces are presented upright. In Fig. 8b, the direction assigned to the face (hair above, chin below) is contrary to the direction assigned to the eyes (eyebrows below, eyes above), but in Fig. 8a, the assigned directions are in coordination. Therefore, we would expect to identify President Bush better in $8 \mathrm{a}$ than in $8 \mathrm{~b}$. A similar prediction may be derived from the present approach, because in $8 \mathrm{~b}$ the eyes are perceived as being strange in the upright position. In this position, then, the predictions of the two approaches do not differ. But the predictions of the two approaches do conflict when the faces are presented inverted. In that position, the directions assigned to the face and the eyes are coordinated in inverted $8 \mathrm{a}$ and uncoordinated in inverted $8 \mathrm{~b}$. Therefore, we would expect to identify President Bush better in inverted $8 \mathrm{a}$ than in inverted $8 \mathrm{~b}$. By contrast, according to the present approach, we would expect to identify President Bush in inverted $8 \mathrm{~b}$ better than in inverted $8 \mathrm{a}$, because in $8 \mathrm{a}$ the inverted eyes are perceived as being upright but odd. That is, when the pictures are inverted, it is easier to identify the president's features in $8 \mathrm{~b}$ than in $8 \mathrm{a}$.

Given the analysis above, we may conclude that the FSI model furnishes an explanation for the face(UI) effect (and for other related phenomena) and that the concept of the scheme is crucial for providing this account. The main ideas of the model's explanation are as follows: A new face stimulus presented upright is processed efficiently by an upright scheme, and there is full compatibility between this scheme and the new face stimulus, whereas when a new face stimulus and parts (eyes and mouth) are presented in the inverted position, inefficiency and incompatibility arise between the stimulus, an inverted scheme, and upright subschemes (see Fig. 3b). As a result, the latter situation generates more errors in perception and recognition than does the former.

The importance of the concept of the scheme can be highlighted by answering the following question: Can one explain the above facial phenomena through an appeal to the hypothesis that upside-down faces are perceived as being strange because their occurrence has been infrequent? The answer is negative. In itself, this hypothesis cannot explain the complex phenomena of perceiving a face and its parts in the upright and inverted positions. For example, when a face is inverted, one does not perceive it as strange. Rather, one perceives it as an inverted face with upright strange eyes and mouth. As another example, when the cat-woman is inverted, one does not perceive it as a strange woman, but as a totally different creature - a cat. Hence, in these and the other cases discussed above, the explanation is based on the use of the appropriate schemes and subschemes.

\section{Part II discussion}

This discussion is divided into six sections: (1) discussion of several theoretical issues connected to the FSI model; (2) a comparison of the FSI model and the configural-processing hypothesis; (3) the FSI model and facial information; (4) the empirical success of the present model in contending with research findings; (5) future research; and (6) conclusions summarizing the major merits of the present model.

\section{Theoretical issues}

Three issues are discussed here. (a) The FSI model assumes that schemes (and prototypes) bestow face-form meaning on a face stimulus. The question that then arises is, how have these constructs themselves acquired meaning that can be bestowed on a face stimulus? (b) Does the assumption that a scheme interprets and organizes the face stimulus mean that the original visual stimulus has been lost? (c) What are the 
relations between the FSI model and the cognitive system for processing visual information?

Schemes and meaning This question is similar to the homunculus argument: We explain one's meaningful perception by assuming the presence in one's head of a small person who bestows meaning on one's perception-an account that starts an infinite regress of explanation. This argument, in my view, is not devastating, as several suggestions contribute toward solving it. Dennett (1979), for example, suggested decomposing the clever homunculus into several very stupid homunculi, each of whom can be replaced by a mechanical operation (for a discussion, see Rakover, 1990). Another possible answer to the question of schemes and meaning is based on the process of learning: While schemes are being constructed (for Stage 2), they are connected by parents, friends, and other educational agents with appropriate networks of names, descriptions, functions, and meanings (e.g., the mother touches a baby's mouth and says, "this is a mouth to eat, kiss, and speak").

Interpretation and organization The FSI model is based on two key concepts: that of the scheme, as it was developed in psychology in the 1980s, and that of the prototype, which has developed in research on faces from the 1990s onward (see Brewer, 1987; Brewer \& Nakamura, 1984; Goldstein \& Chance, 1980; Rakover, 2002; Rhodes \& Leopold, 2011; Rumelhart, 1984). Given this basis, the following comment should be addressed.

According to the literature, the scheme comes into interaction with specific new information-the input information. This interaction, on the one hand, changes the scheme itself, and on the other hand, produces a specific instantiated memory representation that is a combination of the scheme's information and the input information. The problem is that according to this approach, the input information of the stimulus is lost, since the memory trace is a certain combination of the input information and the scheme (see Brewer \& Nakamura, 1984; this was a major critique of F. C. Bartlett's, 1932, scheme theory). In this respect, it is possible to level this criticism against the present model, too, because the information of the new face stimulus has undergone a series of processing stages (e.g., meaning bestowal, interpretation, and organization). Still, I also do not believe that this criticism is devastating. It may be seen as similar to the general critique of the information-processing approach: Due to the modes of information processing that a visual stimulus has undergone, it is hard to know whether the perceptual outcome is veridical (see Rakover, 1990). And indeed, several studies have shown that in certain special cases, perception and memory are not veridical. For example, new events that the individual has not experienced can be implanted in the memory (e.g., Loftus, 1975). Also, as was described above, a number of experiments have reported the prototype effect: Even though the prototype face was not presented at the study stage, participants asserted at the test stage that the face had been presented at study. And, in many interesting cases connected to perceptual illusions and faces presented upside down, the cognitive system fails and creates distortions and errors in perception and memory. Still, it should be emphasized that in almost all cases in normal everyday life, the visual cognitive system is able to process the visual information (faces, objects, landscapes) in a way that fits reality. This, one may speculate, is the result of evolutionary processes that have allowed one to adapt appropriately to the environment; that is, while adaptation is not perfect, it works well in almost all possible situations.

The FSI model and the visual-processing system The FSI model is conceived of as a subsystem in the general cognitive visual system. Thus, one may view Fig. $3 \mathrm{a}$ as a description of a subsystem that deals with the parts in the cognitive visual system that are needed for handling a visual stimulus of a face, and Fig. $3 \mathrm{~b}$ as a description of how the subsystem handles new face stimuli presented in upright and in inverted positions. $^{7}$

The proposition that the FSI model is anchored to the general visual system may be supported by the following three empirical and theoretical observations. First, many empirical findings have exemplified the general importance of schemes in perceiving and remembering different visual and verbal stimuli (e.g., Brewer, 1987; Brewer \& Nakamura, 1984; Rumelhart, 1984). This can also be illustrated by Fig. 9, which exemplifies the organizational and interpretational power of a scenery scheme. In the upright position, the trees are perceived as being reflected in water on which leaves are floating. When Fig. 9 is inverted, a similar picture is perceived, with the leafage floating on what is perceived as air, but not being reflected in what is perceived as water. (One may propose that the air and water exchange places.) When Fig. 9 is rotated on its side, one perceives a huge tree trunk from which symmetrical branches grow outand so, inexplicably, do humans. In this case, the leafage is seen as being part of all of leaves that grow on the branches of the enormous tree.

Second, the processes of the visual system and of the FSI model interact. This is illustrated by the following example. Peterson (1994) showed that the recognition process, which

\footnotetext{
${ }^{7}$ The proposal that the FSI model represents a subsystem in the general visual system assumes a neutral stance in the long-lasting debate between the face-specific-mechanism hypothesis and the expertise hypothesis (see note 3 ). Both the system and the subsystem can be characterized as being based on innate and learned processes, which have generated these adaptive and interactive cognitive structures.
} 


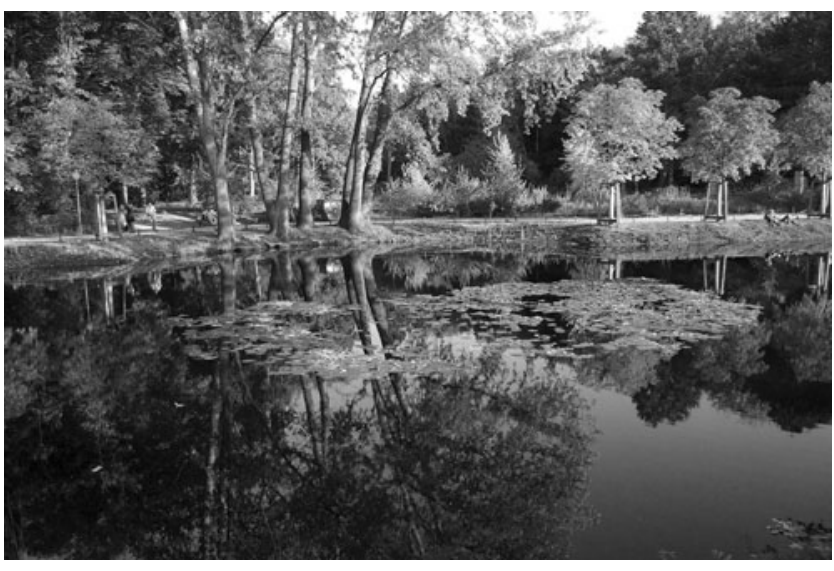

Fig. 9 Water reflection. Upright, the threes are perceived as being reflected in water on which there is leafage. Inverted, a similar picture is perceived with the leafage floating in the air and not reflected in what is perceived as water. From the side, a huge tree trunk is perceived, with symmetrical branches growing out, as well as some humans. (Park Tiergarten, Berlin. The picture was photographed by Maor Dvir and is reproduced with permission.)

is considered a later step in processing visual information, influences the perception of figure/ground, which is considered the outcome of earlier processes. Figure 10 shows a similar process connected to the present model. As may be seen, in Fig. 10b the image of a schematic face (profile) appears, shaded with slanting lines against a background of horizontal lines. But, if we turn the figure upside down, we obtain the image of a schematic face shaded with horizontal lines against a background of slanting lines. In Fig. 10a, such a perceptual phenomenon of figure/ground does not appear. The difference between these two figures (of equal size, texture, and symmetry of the two parts) is that in $10 \mathrm{~b}$, a meaningful form can be discerned: a human profile (even though it is reasonable to assume that the reader has come across this figure for the first time), while in 10a, no meaningful form of this kind is discernible. According to the present model, the scheme of a human profile has conferred face-form meaning on the visual stimulus in $10 \mathrm{~b}$ (which is also why we may see a human face in the clouds).

a
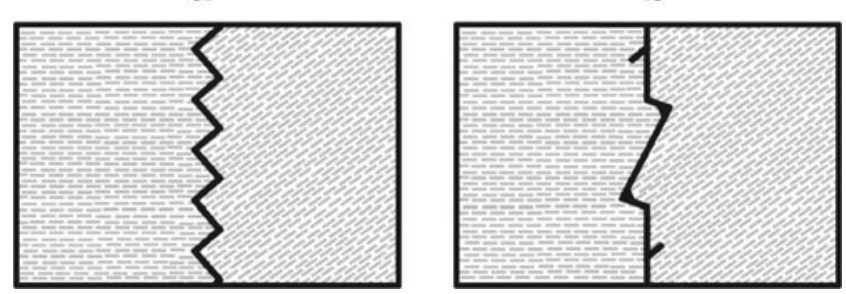

Fig. 10 In panel (b), the profile of a man appears against a background shaded with horizontal lines, while in panel (a), a similar effect of figure and ground does not appear. This effect does not change when the figure is inverted. (Drawings by the author)
Note that the meaning is conferred on Fig. 10b by means of a scheme and not a prototype, because the figure is very different from a real prototype of a profile.

Third, according to Fig. 3, after the comparison of the new face stimulus with the schemes and prototypes and after meaning bestowal, the information undergoes additional processing, such as storage and retrieval. An important question is how is this information stored? The literature suggests two basic approaches: the exemplarbased and the norm-based approaches. The former approach proposes that exemplars of faces are represented in memory separately, and classification or recognition of a new face is accomplished by the use of a rule of similarity between the new face and the faces represented in memory (e.g., Nosofsky \& Johansen, 2000). By contrast, the norm-based approach holds that faces are represented in memory by their deviations from the norm (prototype), and this norm is updated as a result of exposure to new faces (e.g., Rhodes \& Leopold, 2011). The debate between these two approaches is long-lasting. On the one hand, Nosofsky and Johansen were able to show that many effects considered to support the normbased approach can be explained by the exemplar-based approach (see also Hintzman, 1986). On the other, Rhodes and Leopold showed that predictions related to phenomena of perceptual aftereffect that were inferred from the norm-based approach were better than predictions inferred from the exemplar-based approach. (The perceptual aftereffect may be illustrated as follows: If we look at a picture of the face of David in which he is shown as very fat, we will afterward perceive his usual face as thinner, and likewise, if we look at David's face when he has grown exceedingly thin, we will afterward perceive his usual face as fatter.)

Here I want to raise one problem regarding each of these two approaches, and each of which has to do with our concern. It is hard to see how the exemplar-based approach would be able to handle Fig. 10b on the basis of a rule based on the similarity between this figure and thousands of exemplars of real profiles represented in memory. Perhaps the solution lies in the development of a rich theory of similarity, as Nosofsky and Johansen (2000) proposed in a discussion of complicated cases. By contrast, as stated above, it is easy to explain the perception of this figure as a human profile relying on an abstract scheme of a human profile.

On the other hand, if in the norm-based approach a face is coded according to its deviations from the norm, and if the norm undergoes changes as a result of exposure to new faces (an important assumption for explaining perceptualaftereffect phenomena), it follows that all of the faces represented as deviations from the norm must change as the norm changes. This is an uncomfortable conclusion, since it 
does not accord with the everyday observation that we remember a friends' face as we saw it the last time (even after many years). ${ }^{8}$

In view of this discussion, one may suggest that the FSI model differs from these two approaches. It differs from the exemplar-based approach, since a new face stimulus is compared to schemes and prototypes, and it differs from the norm-based approach, since a face is not represented as deviations from a norm but as a meaning-bearing face. While these two approaches are connected primarily to the storage and retrieval stages of information processing, the FSI model is connected to the encoding stage. As mentioned above, after the schemes, subschemes and prototypes have been applied to the face stimulus, the facial information moves on to further processing stages: It is stored in longterm memory and retrieved when needed, in such cases as in a recognition test (See Fig. 3a).

The FSI model has not been developed to include additional cognitive mechanisms for dealing with the storage and retrieval of facial information, since the goal of the present article is to provide answers to the previous two questions and an explanation for the face(UI) effect-goals that are achieved in the encoding stage. Nevertheless, it would be instructive to know how the model might cope with a situation in which it was put to an empirical test that involved the retrieval of facial information (see also the Future Research section). To examine this issue, consider the following proposed recognition experiment (which, to the best of my knowledge, has not been reported).

The experiment, which uses oval faces, "egg-faces" (i.e., faces without hair and ears, in which the focus of one's attention is on the internal facial features), is based on a yes/no recognition procedure. In the study stage, $n$ egg-faces are presented one by one. In the test stage, $2 n$ egg-faces are presented one by one: $n$ old faces randomly mixed with $n$ new faces. The participants have to decide, for each face, whether it is old or new. Four independent groups are run in the test stage. They are differentiated in terms of the division of a face into two parts (in which each part can appear upright or inverted): one is a face* — that is, a face without eyes and mouth - and the other is the eyes and mouth. In the upright/upright group, both face* and the eyes and mouth appear in the upright position. In the upright/inverted group, the face* appears upright and the eyes and mouth upside

\footnotetext{
${ }^{8}$ One possible solution to this problem is to assume that the new facial information is processed in two steps. First, the average between the new faces and the norm is calculated - a calculation that greatly alters the norm for a short time. This calculation, however, does not involve all of the previous faces represented in memory. Second, the new faces are added to all of the previous faces, and the norm - the average - is recalculated - a calculation whose effect on the norm is small. (Note that this problem is not so relevant for the scheme of a face, because of a scheme's abstract nature.)
}

down. In the inverted/upright group, face* appears inverted and the eyes and mouth upright. In the inverted/inverted group, both parts of the face appear upside down.

On the basis of the decision mechanism above that deals with face-scheme incompatibilities, and assuming that recognition increases as a function of the decrease in these incompatibilities (an assumption that connects the response to the model's mechanisms), the following predictions can be made: Recognition in the upright/upright group will be the best, since there are no face-scheme incompatibilities. Recognition in the inverted/upright group will be second best, since the upright eyes and mouth would suit the appropriate upright subschemes, and the inverted face* would be handled by the inverted scheme with low processing efficiency. Recognition in the inverted/inverted group would be third best, since the inverted eyes and mouth would be perceived as upright and strange, and the inverted face* would be handled by an inverted scheme. And recognition in the upright/inverted group would be the worst, since the inverted eyes and mouth would be perceived as upright and strange, and the upright face* would be handled by an upright efficient scheme, which would increase the two strange features' saliency. This prediction has received indirect and partial support from experiments that differed from the one proposed here (e.g., Boutsen \& Humphreys, 2003; Rakover, 1999; Rhodes et al., 1993). Finally, note that according to the configural-processing hypothesis, recognition should be better in the upright/inverted group than in the inverted/inverted group, since in the latter group the configural-information processing of the whole face would be impaired, whereas in the former group impairment would be restricted to the inverted eyes and mouth.

\section{FSI model and the configural-processing hypothesis}

The explanation proposed by the FSI model for the face(UI) effect is different from the one proposed by the configuralprocessing hypothesis. While the latter suggests that errors in the perception and recognition of inverted face are caused by impairment in the processing of configural information, the FSI model suggests that they are caused by the processes of matching schemes and subschemes to a face stimulus and its parts, respectively.

Despite this difference between the two explanations, the impairment of configural information caused by inversion can be seen as being accounted for by the FSI model. Interpretational and organizational differences distinguish the processing of inverted and an upright faces and facial features. Inverted eyes are perceived as being upright and strange, since the eyes' components and their spatial relations (i.e., their configural information) are processed (interpreted and organized) so as to accord with an upright subscheme. Hence, while the configural-processing 
hypothesis assumes the existence of a causal relation between inversion and impairment in processing configural information, the FSI model suggests an explanation for this relation: the process of matching schemes and subschemes to a face stimulus and its parts. In this respect, inversion may be said to impair the processing of configural information since the information (componential and configural) of the inverted feature (e.g., eyes) is interpreted and organized in terms of an upright subscheme, when the rest of the face is handled by an inefficient inverted scheme. I do not propose here that the configural-processing hypothesis can be reduced to the FSI model (in the sense that the former is deduced from the latter, together with certain bridging laws; see Rakover, 1990, 2007). Rather, the present model (a) provides an explanatory basis for the configural-processing hypothesis and (b) explains what this hypothesis accounts for, and moreover is able to elucidate additional phenomena that the hypothesis is unable to explain.

I shall illustrate this difference with two additional examples (for other examples, see the Several Illustrations section above). The first shows that a given phenomenon that was explained by the configural-processing hypothesis can be accounted for differently by the FSI model, and the second shows that a certain phenomenon can be explained better by the FSI model than by the configural-processing hypothesis.

The first example concerns the finding that the transition from the upright to the inverted position $\left(0^{\circ}\right.$ to $180^{\circ}$ ) is not monotonic. According to the configuralprocessing hypothesis, nonmonotonicity is explained by the qualitative change in processing information: from featural and configural to mainly featural. According to the present approach, nonmonotonicity is explained by the efficiency assumption discussed above; that is, the steep decline in the frequency of face stimuli presented at an angle greater than about $90^{\circ}$, which results in the generation of inefficient inverted schemes.

The second example is illustrated in Fig. 11, which demonstrates the difference between these two explanations. In both Fig. 11a and b, the eyes are tilted by the same angular amount $\left(20^{\circ}\right)$; that is, the sizes of the configural change are equal. Nevertheless, the perceptual meaning of these faces is different: While Fig. 11a shows the image of a Chinese person with a neutral expression, Fig. 11b shows the image of a Caucasian person with a downcast expression. It is hard to explain this perceptual difference by means of the configural-processing hypothesis, because the sizes of the configural change are the same, so one needs additional information to explain the difference in the perception of these faces. This additional information is given by the schemes of the white person and the Chinese person. A similar explanation is provided when Fig. 11 is inverted: While the inverted Fig. $11 \mathrm{~b}$ will be perceived as an image of
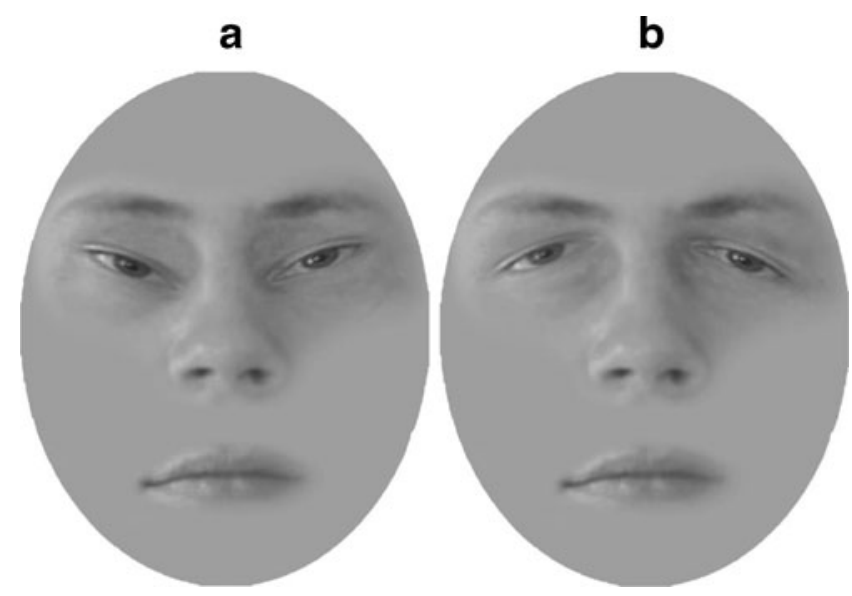

Fig. 11 Perceptions of face distortions of equal magnitude: (a) a Chinese person with a neutral expression, and (b) a Caucasian with a miserable expression, created by the same level of configural change (eyes skewed by the same angular amount, $20^{\circ}$ )

a Chinese person, one may tend to perceive the inverted Fig. 11a as an image of a Caucasian person.

\section{FSI model and facial information}

To understand how the present model handles facial information, recall that the model represents a subsystem that is included in the general visual system. Both of these deal with the visual information of a face that is presented in a certain experimental situation, whose processing is guided by a particular task. I shall discuss this situation here and in the following section.

To recapitulate, according to the FSI model, matching the schemes and subschemes to an inverted face and inverted parts differentially disrupts the facial information (featural information, first-order relational properties, configural information or second-order relational properties, and holistic information of the whole face stimulus). Because of this, holistic processing and the bestowing of face-form meaning on a whole face are disrupted. Thus, the FSI model may be viewed as providing an explanatory basis for both the holistic hypothesis and Rossion's $(2008,2009)$ hypothesis, which proposes the constriction of the perceptual field under inversion. As a result of the attempts to match schemes and subschemes to the inverted face stimulus and its parts, attention is constricted to local areas in the face, such as the inverted eyes and mouth, which are perceived as being upright but weird.

Attention has important effects on processing inverted faces. Inversion has a minimal or no effect on a facial features within the context of a whole face when a change made to that feature's color but not its shape (e.g., blackening the mouth or some of the teeth; see McKone \& Yovel, 2009). In these cases, the visual system processes seem to 
take over, the feature becomes salient-an attention attractor-and the effect of inversion disappears. Similarly, Rakover (2012) found that perception and recognition of the eyes (within the whole face or in isolation) in upright and inverted positions were the same when the irises were blackened. In the same vein, focusing attention on a local area in the face reduces the effect of inversion (e.g., Barton et al., 2001; Tanaka \& Gordon, 2011).

Given these facts, the second question above arises here: How does the present model handle the common approach (proposed by the configural-processing hypothesis) that inversion impairs mainly the processing of configural information? The answer is that the present model proposes that inversion has multiple disruptive effects (which are also dependent on experimental manipulations and tasks; see the Discussion in the Controversy Between the Configural-Processing and Holistic Hypotheses section above). For example, the model suggests that both of these kinds of information -featural and configural-are affected when inverted eyes are perceived as strange upright eyes (see Fig. 7). In this respect, I agree with McKone and Yovel's (2009) conclusion from their literature review: "The results clearly reject the widespread view that feature inversion effects are typically only small, and much smaller than spacing inversion effects" (p. 795; see the Findings That Do Not Support the Configural-Processing Hypothesis section above).

\section{Empirical success}

From the description and discussion of the FSI model, it is evidently able to handle many findings regarding the effect of inversion on face processing and to propose an explanatory basis for the configural-processing and the holistic hypotheses. An important reason for this is that the model handles the above-mentioned four kinds of facial information (i.e., featural information, first-order relational properties, configural information or secondorder relational properties [between and within features], and holistic information).

Nevertheless, the following question has to be tackled in particular: How does the present model contend with experimental findings attesting that configural information is neither a necessary nor a sufficient condition for the face (UI) effect?

First, I shall examine the question of necessity. The findings that have shown that inversion also impairs processing of isolated features (e.g., hair and forehead, eyes) have emphasized the importance of spatial relations within the features no less than their importance between features. These findings, even though they do not support the configural-processing hypothesis, accord with the present model: Some of the inverted isolated facial features (e.g., the components and the spatial relations of the eyes) are handled by upright subschemes and are thus perceived as upright and strange, and some (hair and forehead, nose, and facial outline) are handled by an inverted scheme and subschemes and are processed with low efficiency.

I now move on to examine the question of sufficiency. The finding that inversion does not impair processing of the configural information connected to the eye illusion does not confirm the configural-processing hypothesis. How does the present model tackle this finding? The answer lies in the fact that the model depicts a subsystem of the general visual system, which deals with any kind of visual stimuli. In this respect, a face stimulus is treated as both (a) a visual stimulus, subject to general perceptual processes that in certain conditions are also responsible for the creation of geometrical illusions (see Coren \& Girgus, 1978; Robinson, 1998), and (b) a face stimulus, treated by means of processes intended to deal with stimuli of this kind (e.g., by comparison of a given face stimulus with facial schemes, subschemes, and prototypes). It seems that the eye illusion is produced mainly by the general visual system, and as a result the eye illusion functions like a geometrical illusion. The reason for the absence of an inversion effect on this illusion apparently lies in its geometrical symmetry. This illusion is constructed by a $20 \%$ increase (or decrease) in size of the whole face except for the eye area. The increase creates proportional configural changes similar to the symmetrical spatial changes connected to Ebbinghaus's/Titchener's geometrical illusion (i.e., this illusion is also unaffected by inversion). By contrast, an increase of the intereye distance or the nose-mouth distance creates configural changes that are not proportional: In the first case, the distance between the eyes and the cheek contour decreases relatively more than the distance between the eyes and the chin contour, and likewise the change in nose-mouth distance enlarges the distance between the mouth and the eyes more than the distance between the mouth and the cheek contour.

\section{Future research}

The FSI model gives rise to the following three relatively new questions (the first two are empirical, and the third is theoretical) that concern the concept of the face scheme.

What about the face stimulus's components and their organization is essential to activate the appropriate scheme (or subscheme)? Some of the illustrations discussed above exemplify that different, and sometimes similar, components 
are involved in activating different schemes and subschemes in upright and inverted positions. For example, the inverted Fig. 5 illustrates that a cat scheme and subschemes may be activated by the following features: ears, nose, whiskers, and mouth. However, these features are not those needed for the activation of the woman's face scheme, and Fig. 7 illustrates that the major components and their spatial relations (a pupil between the eyelids) may be responsible for the activation of the eye subscheme in both upright and inverted positions. These illustrations suggest the following general research program as a possible answer to the question above.

By experimental manipulation, certain components can be removed from (or added to) a visual stimulus to examine whether the appropriate scheme (or subscheme) is still activated. The manipulation can be incorporated into several experimental procedures, such as priming. To exemplify this idea, consider Fig. 12. The two dots in the three illustrations are perceived as representing eyes. However, when Fig. 12 is inverted, the perception changes: The dots with the curved line in Fig. 12a are perceived as a mouth, whereas in Fig. $12 \mathrm{~b}$ and $\mathrm{c}$ they are still perceived as eyes. This leads to the following three interesting tentative conclusions: (a) The location of the dots in the inverted Fig. 12b is not a strong enough cue for perceiving them as a mouth; (b) the curved line connecting the dots in the inverted Fig. 12a cause them to be perceived as a mouth; and (c) the appearance of the nose and mouth in the inverted Fig. 12c weaken the tendency to perceive the dots with the curved line as a mouth. (This kind of research can be supported by the research on Pareidolia, which deals with the tendency to see human faces in certain visual forms; e.g., Chalup, Hong, \& Ostwald, 2010.)

What are the relations between a face scheme and its face subschemes? Navon (1977) showed that under certain conditions, the global properties of a given stimulus determine its percept. Could such a relation be an answer to question in

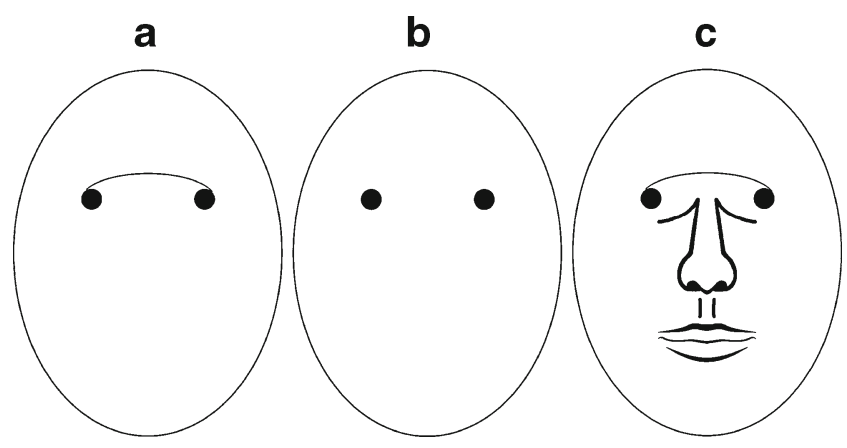

Fig. 12 The two dots in the three illustrations are perceived as eyes. When the figure is inverted, the dots with the curved line in panel (a) are perceived as a mouth, whereas in panels (b) and (c) they are still perceived as eyes and not as a mouth. (Drawings by the author) this section? Figure 13, a "one face or two" stimulus, portrays a much more complex answer. When shifting one's gaze at the figure, one oscillates between perceiving it as one global face and two local faces. This is probably caused by a highly efficient upright face scheme(s). However, when the figure is inverted, this oscillation disappears, and the four black circles are perceived sometimes as car headlights or sometimes as two pairs of eyes above a smiling mouth. These may be accounted for by an appeal to the assumption that the inverted face scheme has low processing efficiency and the activation of other, upright subschemes.

Can a face scheme be conceived of as a mechanism? An affirmative answer would rest on the following analogy: A face scheme is constituted of certain facial components (facial subschemes that handle facial features) that interact (the spatial relations among the subschemes) so as to generate the phenomenon under investigation (perception of faces in upright and inverted positions). If this analogy holds, facial schemes (and subschemes) may be viewed as certain perceptual mechanisms interlinked in the general system for processing visual information. Nevertheless, there seems to be an interesting difference between a mechanical mechanism, such as a flashlight, and a face scheme, which may suggest a negative answer to this question. While the light in the flashlight is turned on by the operation of a switch, the activation of a face scheme is not initiated by such a trigger, but by a match between certain components in the face stimulus and

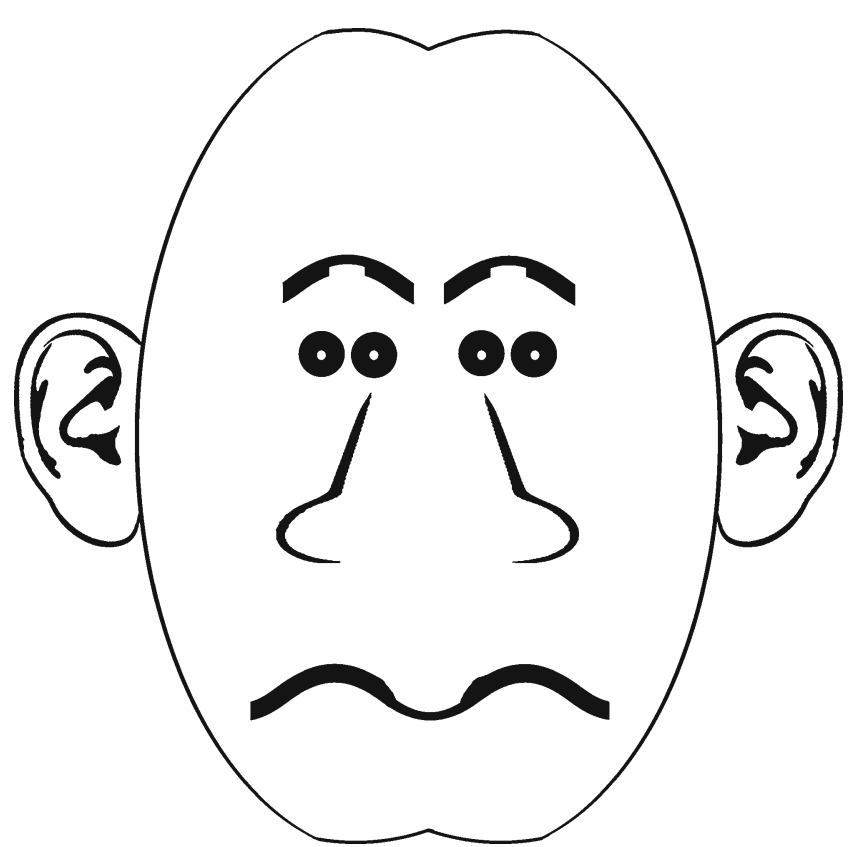

Fig. 13 "One face or two" (drawing by the author). Perception of the figure oscillates between one global face and two local faces. When the figure is inverted, the oscillation disappears 
similar components in the scheme (when a successful match is determined by the criterion of applicability).

\section{Conclusion}

The model is notable for the following three important ideas:

- The FSI model is conceived as depicting a subsystem of the visual cognitive system, and a visual stimulus of a face is treated by both system and subsystem.

- The model explains how the cognitive constructs of schemes and prototypes are generated; these constructs are needed to explain how a face stimulus is transformed into a meaning-bearing face.

- The model explains how the schemes and prototypes deal with a face and its parts presented upright and inverted, thereby offering an explanation for the face(UI) effect, answering the questions of how faces are recognized and why inversion seems to impair configural information, and providing an explanatory basis for the configuralprocessing and holistic hypotheses.

Author note I am grateful to Shmuel Ahituv, Maor Dvir, and Rinat Philipp for the preparation of the figures. I am indebted to the editor, C. Moore, and to two anonymous reviewers for their valuable comments.

\section{References}

Amishav, R., \& Kimchi, R. (2010). Perceptual integrality of componential and configural information in faces. Psychonomic Bulletin \& Review, 17, 743-748.

Bailer-Jones, D. M. (2009). Scientific models in philosophy of science. Pittsburgh, PA: University of Pittsburgh Press.

Bartlett, F. C. (1932). Remembering. Cambridge, UK: Cambridge University Press.

Bartlett, J. C., \& Searcy, J. H. (1993). Inversion and configuration of faces. Cognitive Psychology, 25, 281-316.

Bartlett, J. C., Searcy, J. H., \& Abdi, H. (2003). What are the routes to face recognition? In M. A. Peterson \& G. Rhodes (Eds.), Perception of faces, objects, and scenes: Analytic and holistic processes (pp. 21-52). Oxford, UK: Oxford University Press.

Barton, J. J. S., Keenan, J. P., \& Bass, T. (2001). Discrimination of spatial relations and features in faces: Effects of inversion and viewing duration. British Journal of Psychology, 92, 527-549.

Baudouin, J.-Y., \& Humphreys, G. W. (2006). Configural information in gender categorization. Perception, 35, 531-540.

Bechtel, W. (2008). Mental mechanisms: Philosophical perspectives on cognitive neuroscience. New York, NY: Routledge.

Bechtel, W., \& Wright, C. D. (2009). What is psychological explanation? In J. Symons \& P. Calvo (Eds.), The Routledge companion to philosophy of psychology (pp. 113-130). London, UK: Routledge.

Biederman, I. (1987). Recognition-by-components: A theory of human image understanding. Psychological Review, 94, 115-147. doi:10.1037/0033-295X.94.2.115
Biederman, I., \& Kalocsai, P. (1998). Neural and psychophysical analysis of object and face recognition. In H. Wechsler, P. J. Phillips, V. Bruce, F. Fogelman Soulié, \& T. S. Huang (Eds.), Face recognition: From theory to application (pp. 3-25). Berlin, Germany: NATO Scientific Affairs Division and Springer.

Boutsen, L., \& Humphreys, G. W. (2003). The effect of inversion on the encoding of normal and "thatcherized" faces. Quarterly Journal of Experimental Psychology, 56A, 955-975. doi:10.1080/02724980244000774

Brewer, W. F. (1987). Schemas versus mental models in human memory. In P. Morris (Ed.), Modelling cognition (pp. 187-197). Chichester, UK: Wiley.

Brewer, W. F., \& Nakamura, G. V. (1984). The nature and functions of schemas. In R. S. Wyer Jr. \& T. K. Srull (Eds.), Handbook of social cognition (Vol. 1, pp. 119-160). Hillsdale, NJ: Erlbaum.

Bruyer, R. (2011). Configural face processing: A meta-analytic survey. Perception, 40, 478-1490.

Burton, A. M., \& Jenkins, R. (2011). Unfamiliar face perception. In A. J. Calder, G. Rhodes, M. H. Johnson, \& J. V. Haxby (Eds.), The Oxford handbook of face perception (pp. 287-306). Oxford, UK: Oxford University Press.

Cabeza, R., \& Kato, T. (2000). Features are also important: Contributions of featural and configural processing to face recognition. Psychological Science, 195, 312-314.

Calder, A. J., Young, A. W., Keane, J., \& Dean, M. (2000). Configural information in facial expression perception. Journal of Experimental Psychology. Human Perception and Performance, 26, 527-551. doi:10.1037/0096-1523.26.2.527

Carbon, C.-C., \& Leder, H. (2005). When feature information comes first! Early processing of inverted faces. Perception, 34, 117-134.

Chalup, S. K., Hong, K., \& Ostwald, M. J. (2010). Simulating Pareidolia of faces for architectural image analysis. International Journal of Computer Information Systems and Industrial Management Applications, 2, 262-278.

Collishaw, S. M., \& Hole, G. J. (2000). Featural and configural processes in the recognition of faces of different familiarity. Perception, 29, 893-909.

Coren, S., \& Girgus, J. S. (1978). Seeing is deceiving: The psychology of visual illusions. Hillsdale, NJ: LEA.

Davidoff, J., \& Donnelly, N. (1990). Object superiority: A comparison of complete and part probs. Acta Psychologica, 73, 225-243.

Dennett, D. (1979). Brainstorms. Hassocks, UK: Harvester Press.

Diamond, R., \& Carey, S. (1986). Why faces are and are not special: An effect of expertise. Journal of Experimental Psychology. General, 115, 107-117.

Donnelly, N., \& Davidoff, J. (1999). The mental representations of faces and houses: Issues concerning parts and wholes. Visual Cognition, 6, 319-343.

Estes, W. K. (1994). Classification and cognition. New York, NY: Oxford University Press.

Fabiani, M., \& Donchin, E. (1995). Encoding processes and memory organization: A model of the von Restorff effect. Journal of Experimental Psychology: Learning, Memory, and Cognition, 21, 224-240. doi:10.1037/0278-7393.21.1.224

Fagan, M. B. (2012). The joint account of mechanistic explanation. Philosophy of Science, 79, 448-472.

Farah, M. J. (1992). Is an object an object? Cognitive and neuropsychological investigations of domain specificity in visual object recognition. Current Directions in Psychological Science, 1, 164 169.

Farah, M. J., Tanaka, J. W., \& Drain, H. M. (1995). What causes the face inversion effect? Journal of Experimental Psychology. Human Perception and Performance, 21, 628-634.

Farah, M. J., Wilson, K.-D., Drain, M., \& Tanaka, J. W. (1998). What is "special" about face perception? Psychological Review, 105, 482-498. doi:10.1037/0033-295X.105.3.482 
Freire, A., Lee, K., \& Symons, L. A. (2000). The face-inversion effect as a deficit in the encoding of configural information: Direct evidence. Perception, 29, 159-170.

Gauthier, I., \& Bukach, C. (2007). Should we reject the expertise hypothesis? Cognition, 103, 322-330. doi:10.1016/ j.cognition.2006.05.003

Gauthier, I., \& Tarr, M. J. (1997). Becoming a "Greeble" expert: Exploring mechanisms for face recognition. Vision Research, 37, 1673-1682.

Goffaux, V., \& Rossion, B. (2007). Face inversion disproportionately impairs perception of vertical but not horizontal relations between features. Journal of Experimental Psychology. Human Perception and Performance, 33, 995-1001.

Gold, J. M., Mundy, P. J., \& Tjan, B. S. (2012). The perception of a face is no more than the sum of its parts. Psychological Science, $23,427-434$.

Goldstein, A. G., \& Chance, J. E. (1980). Memory for faces and schema theory. Journal of Psychology, 105, 47-59.

Hintzman, D. L. (1986). "Schema abstraction" in multiple-trace memory model. Psychological Review, 93, 411-423. doi:10.1037/ 0033-295X.93.4.411

Hummel, J. E. (2003). The complementary properties of holistic and analytic representations of shapes. In M. A. Peterson \& G. Rhodes (Eds.), Perception of faces, objects, and scenes: Analytic and holistic processes (pp. 212-234). Oxford, UK: Oxford University Press.

Ingvalson, E. M., \& Wenger, M. J. (2005). A strong test of dual-mode hypothesis. Perception \& Psychophysics, 67, 14-35.

Kemp, R., McManus, C., \& Pigott, T. (1990). Sensitivity to the displacement on the perception of facial features in negative and inverted images. Perception, 19, 531-543.

Kim, J. (2011). Philosophy of mind (3rd ed.). Boulder, CO: Westview Press.

Kimchi, R., \& Amishav, R. (2010). Faces as perceptual wholes: The interplay between component and configural properties in face processing. Visual Cognition, 18, 1034-1062.

Leder, H., \& Bruce, V. (1998a). Feature processing from upright and inverted faces. In H. Wechsler, P. J. Phillips, V. Bruce, F. Fogelman Soulié, \& T. S. Huang (Eds.), Face recognition: From theory to applications (pp. 547-555). Berlin, Germany: NATO Scientific Affairs Division and Springer.

Leder, H., \& Bruce, V. (1998b). Local and relational aspects of face distinctiveness. Quarterly Journal of Experimental Psychology, 51A, 449-473.

Leder, H., \& Bruce, V. (2000). When inverted faces are recognized: The role of configural information in face recognition. Quarterly Journal of Experimental Psychology, 53A, 513-536.

Leder, H., Candrian, G., Huber, O., \& Bruce, V. (2001). Configural features in the context of upright and inverted faces. Perception, 30, 73-83.

Leder, H., \& Carbon, C. C. (2004). Part-to-whole effects and configural processing in faces. Psychology Science, 46, 531-543.

Leder, H., \& Carbon, C. C. (2005). When context hinders! Learn-test compatibility in face recognition. Quarterly Journal of Experimental Psychology, 58A, 235-250.

Leder, H., \& Carbon, C. C. (2006). Face-specific configural processing of relational information. British Journal of Psychology, 97, 19-29.

Lee, K., Anzures, G., Quinn, P. C., Pascalis, O., \& Slater, A. M. (2011). Development of face processing expertise. In A. J. Calder, G. Rhodes, M. H. Johnson, \& J. V. Haxby (Eds.), The Oxford handbook of face perception (pp. 753-778). Oxford, UK: Oxford University Press.

Liu, J., Harris, A., \& Kanwisher, N. (2002). Stages of processing in face perception: An MEG study. Nature Neuroscience, 5, 910 916

Loftus, E. F. (1975). Leading questions and the eyewitness report. Cognitive Psychology, 7, 560-572.
Loftus, G. R., Oberg, M. A., \& Dillon, A. M. (2004). Linear theory, dimensional theory, and the face-inversion effect. Psychological Review, 111, 835-863.

Maco, S., \& Leder, H. (1988). Your eyes only? A test of interactive influence in the processing of facial features. Journal of Experimental Psychology. Human Perception and Performance, 24, 1486-1500.

Mäntylä, T., \& Holm, L. (2005). Remembering parts and wholes: Configural processing in face recollection. European Journal of Cognitive Psychology, 17, 753-769.

Marr, D. (1982). Vision. San Francisco, CA: Freeman.

Maurer, D., Le Grand, R., \& Mondloch, C. J. (2002). The many faces of configural processing. Trends in Cognitive Sciences, 6, 255260.

McGinn, C. (1991). The problem of consciousness: Essays towards a resolution. Oxford, UK: Blackwell.

McKone, E. (2010). Face and object recognition: How do they differ? In V. Coltheart (Ed.), Tutorials in visual cognition (pp. 261-303). Hove, UK: Psychology Press.

McKone, E., Crookes, K., \& Kanwisher, N. (2009). The cognitive and neural development of face recognition in humans. In M. S. Gazzaniga (Ed.), The cognitive neurosciences (4th ed., pp. 467482). Cambridge, MA: MIT Press, Bradford Books.

McKone, E., Martini, P., \& Nakayama, K. (2003). Isolating holistic processing in faces (and perhaps objects). In M. A. Peterson \& G. Rhodes (Eds.), Perception of faces, objects, and scenes: Analytic and holistic processes (pp. 92-119). Oxford, UK: Oxford University Press.

McKone, E., \& Peh, Y. X. (2006). Memory conjunction errors for realistic faces are consistent with configural processing. Psychonomic Bulletin \& Review, 13, 106-111. doi:10.3758/ BF03193820

McKone, E., \& Robbins, R. (2007). The evidence rejects the expertise hypothesis: Reply to Gauthier \& Bukach. Cognition, 103, 331336. doi:10.1016/j.cognition.2006.05.014

McKone, E., \& Robbins, R. (2011). Are faces special? In A. J. Calder, G. Rhodes, M. H. Johnson, \& J. V. Haxby (Eds.), The Oxford handbook of face perception (pp. 149-176). Oxford, UK: Oxford University Press.

McKone, E., \& Yovel, G. (2009). Why does picture-plane inversion sometimes dissociate perception of features and spacing in faces, and sometimes not? Toward a new theory of holistic processing. Psychonomic Bulletin \& Review, 16, 778-797. doi:10.3758/ PBR.16.5.778

Medin, D. L., \& Schaffer, M. M. (1978). Context theory of classification learning. Psychological Review, 85, 207-238. doi:10.1037/ 0033-295X.85.3.207

Mondloch, C., Le Grand, R., \& Maurer, D. (2002). Configural face processing develops more slowly than featural face processing. Perception, 31, 553-566.

Murray, J. E., Rhodes, G., \& Schuchinsky, M. (2003). When is a face not a face? The effects of misorientation on mechanisms of face perception. In M. A. Peterson \& G. Rhodes (Eds.), Perception of faces, objects, and scenes: Analytic and holistic processes (pp. 75-91). Oxford, UK: Oxford University Press.

Murray, J. E., Yong, E., \& Rhodes, G. (2000). Revisiting the perception of upside-down faces. Psychological Science, 11, 492-496.

Navarro, D. J., \& Lee, M. D. (2004). Common and distinctive features in stimulus similarity: A modified version of the contrast model. Psychonomic Bulletin \& Review, 11, 961-974. doi:10.3758/ BF03196728

Navon, D. (1977). Forest before trees: The precedence of global features in visual processing. Cognitive Psychology, 9, 353-383. doi:10.1016/0010-0285(77)90012-3

Nosofsky, R. M. (1984). Choice, similarity, and the context theory of classification. Journal of Experimental Psychology: Learning, 
Memory, and Cognition, 10, 104-114. doi:10.1037/02787393.10.1.104

Nosofsky, R. M. (1992). Similarity scaling and cognitive process models. Annual Review of Psychology, 43, 25-53.

Nosofsky, R. M., \& Johansen, M. K. (2000). Exemplar-based accounts of "multiple-system" phenomena in perceptual categorization. Psychonomic Bulletin \& Review, 7, 375-402.

Palermo, R., \& Rhodes, G. (2002). The influence of divided attention on holistic face perception. Cognition, 82, 225-257.

Palmer, S. E. (2003). Perceptual organization and grouping. In R. Kimchi, M. Behrmann, \& C. R. Olson (Eds.), Perceptual organization in vision: Behavioral and neural perspectives (pp. 3-44). Mahwah, NJ: Erlbaum.

Palmer, S. E., Brooks, J. L., \& Nelson, R. (2003). When does grouping happen? Acta Psychologica, 114, 311-330.

Parks, T. E., Coss, R. G., \& Coss, C. S. (1985). Thatcher and the Cheshire cat: Context and the processing of facial features. Perception, 14, 747-754.

Penry, J. (1971). Looking at faces and remembering them: A guide to facial identification. London, UK: Black Books.

Peterson, M. A. (1994). Object recognition processes can and do operate before figure-ground organization. Current Directions in Psychological Science, 3, 105-111.

Peterson, M. A., \& Rhodes, G. (2003). Introduction: Analytic and holistic processing-The view through different lenses. In M. A. Peterson \& G. Rhodes (Eds.), Perception of faces, objects, and scenes: Analytic and holistic processes (pp. 3-20). Oxford, UK: Oxford University Press.

Rakover, S. S. (1990). Metapsychology: Missing links in behavior, mind and science. New York, NY: Solomon/Paragon.

Rakover, S. S. (1999). Thompson's Margaret Thatcher illusion: When inversion fails. Perception, 28, 1127-1230.

Rakover, S. S. (2002). Featural vs. configurational information in faces: A conceptual and empirical analysis. British Journal of Psychology, 93, 1-30.

Rakover, S. S. (2007). To understand a cat: Methodology and philosophy. Amsterdam/Philadelphia: John Benjamins.

Rakover, S. S. (2008). Is facial beauty an innate response to the Leonardian Proportion? Empirical Studies of the Arts, 26, 155-179.

Rakover, S. S. (2011a). The configural processing hypothesis and the face-inversion effect. In Y.-J. Zhang (Ed.), Advances in face image analysis: Techniques and technologies (pp. 316-333). Hershey, PA: IGI Global. doi:10.4018/978-1-61520-991-0.ch017

Rakover, S. S. (2011b). A plea for methodological dualism and multiexplanation framework in psychology. Behavior and Philosophy, $39,17-43$.

Rakover, S. S. (2012). A feature-inversion effect: Can an isolated feature show behavior like the face-inversion effect? Psychonomic Bulletin \& Review, 19, 617-624.

Rakover, S. S., \& Cahlon, B. (1989). To catch a thief with recognition test: The model and some empirical results. Cognitive Psychology, 21, 423-468.

Rakover, S. S., \& Cahlon, B. (2001). Face recognition: Cognitive and computational processes. Amsterdam, The Netherlands: John Benjamins.

Rakover, S. S., \& Cahlon, B. (2008). A face recognition by similarity (FRBS) conjecture. Perception \& Psychophysics, 70, 969-982. doi:10.3758/PP.70.6.982

Rakover, S. S., \& Cahlon, B. (in press). Cognitive processing of scrambled faces. American Journal of Psychology.

Rakover, S. S., \& Teucher, B. (1997). Facial inversion effects: Part and whole relationship. Perception \& Psychophysics, 59, 752-761.

Reinitz, M. T., Morrissey, J., \& Demb, J. (1994). Role of attention in face encoding. Journal of Experimental Psychology: Learning, Memory, and Cognition, 20, 161-168. doi:10.1037/02787393.20.1.161
Rhodes, G., Brake, K., \& Atkinson, A. (1993). What's lost in inverted faces? Cognition, 47, 25-57.

Rhodes, G., \& Leopold, D. A. (2011). Adaptive norm-based coding of face identity. In A. J. Calder, G. Rhodes, M. H. Johnson, \& J. V. Haxby (Eds.), The Oxford handbook of face perception (pp. 263 286). Oxford, UK: Oxford University Press.

Riesenhuber, M., Jarudi, I., Gilad, S., \& Sinha, P. (2004). Face processing in humans is compatible with a simple shape-based model of vision. Proceedings of the Royal Academy B, 271, S448-S450.

Robbins, R., \& McKone, E. (2007). No face-like processing for objects-of-expertise in three behavioural tasks. Cognition, 103, 34-79.

Robinson, J. O. (1998). The psychology of visual illusion. New York, NY: Dover.

Rock, I. (1973). Orientation and form. New York, NY: Academic Press.

Rock, I. (1974). The perception of disoriented figures. Scientific American, 230, 78-85.

Rock, I. (1988). On Thompson's inverted-face phenomenon. Perception, 17, 815-817.

Rosch, E., \& Mervis, C. B. (1975). Family resemblance: Studies in the internal structure of categories. Cognitive Psychology, 7, 573605. doi:10.1016/0010-0285(75)90024-9

Rossion, B. (2008). Picture-plane inversion leads to qualitative changes of face perception. Acta Psychologica, 128, 274-289.

Rossion, B. (2009). Distinguishing the cause and consequence of face inversion: The perceptual field hypothesis. Acta Psychologica, 132, 300-312.

Rossion, B., \& Gauthier, I. (2002). How does the brain process upright and inverted faces? Behavioral and Cognitive Neuroscience Reviews, 1, 63-75.

Rumelhart, D. E. (1984). Schemata and cognitive system. In R. S. Wyer Jr. \& T. K. Srull (Eds.), Handbook of social cognition, vol. 1 (pp. 161-188). Hillsdale, NJ: Erlbaum.

Schwaninger, A., \& Mast, F. W. (2005). The face-inversion effect can be explained by the capacity limitations of an orientation normalization mechanism. Japanese Psychological Research, 47, 216222.

Schwaninger, A., Ryf, S., \& Hofer, F. (2003). Configural information is processed differently in perception and recognition of faces. Vision Research, 43, 1501-1505.

Searcy, J. H., \& Bartlett, J. C. (1996). Inversion and processing of component and spatial-relational information in faces. Journal of Experimental Psychology. Human Perception and Performance, 22, 904-915.

Sekuler, A. B., Gaspar, C. M., Gold, J. M., \& Bennett, P. J. (2004). Inversion leads to quantitative, not qualitative, changes in face processing. Current Biology, 14, 391-396.

Sekunova, A., \& Barton, J. J. S. (2008). The effects of face inversion on the perception of long-range and local spatial relations in eye and mouth configuration. Journal of Experimental Psychology. Human Perception and Performance, 34, 1129-1135.

Solso, R. L., \& McCarthy, J. E. (1981). Prototype formation of faces: A case of pseudo-memory. British Journal of Psychology, 72, 499503.

Tanaka, J. W., \& Farah, M. J. (1993). Parts and their configuration in face recognition. Quarterly Journal of Experimental Psychology, $46 A, 225-245$.

Tanaka, J. W., \& Farah, M. J. (2003). Perception of faces, objects, and scenes: Analytic and holistic processes. In M. A. Peterson \& G. Rhodes (Eds.), Perception of faces, objects, and scenes: Analytic and holistic processes (pp. 53-74). Oxford, UK: Oxford University Press. doi:10.1093/acprof:oso/9780195313659.003.0003

Tanaka, J. W., \& Gordon, I. (2011). Features, configuration, and holistic face processing. In A. J. Calder, G. Rhodes, M. H. Johnson, \& J. V. Haxby (Eds.), The Oxford handbook of face 
perception (pp. 177-194). Oxford, UK: Oxford University Press. doi:10.1093/oxfordhb/9780199559053.013.0010

Tanaka, J. W., \& Sengco, J. A. (1997). Features and their configuration in face recognition. Memory \& Cognition, 25, 583-592.

Thompson, P. (1980). Margaret Thatcher: A new illusion. Perception, 9, 483-484.

Tulving, E. (1983). Memory and consciousness. Canadian Psychology, 26, $1-12$.

Tversky, A. (1977). Features of similarity. Psychological Review, 84, $327-352$.

Valentine, T. (1988). Upside-down faces: A review of the effect of inversion on face recognition. British Journal of Psychology, 79, 471-491.

Valentine, T. (1991). A unified account of the effects of distinctiveness, inversion and race in face recognition. Quarterly Journal of Experimental Psychology, 43A, 161-204.

Valentine, T., \& Bruce, V. (1988). Mental rotation of faces. Memory \& Cognition, 16, 556-666.

Wright, C. D., \& Bechtel, W. (2007). Mechanisms and psychological explanation. In P. Thagard (Ed.), Philosophy of psychology and cognitive science (pp. 31-77). Amsterdam, The Netherlands: Elsevier.

Wright, D. B., Boyd, C. E., \& Tredoux, C. G. (2003). Inter-racial contact and the own-race bias for face recognition in south Africa and England. Applied Cognitive Psychology, 17, 365-373.

Yin, R. K. (1969). Looking at upside-down faces. Journal of Experimental Psychology, 81, 141-145.

Young, A. W., Hellawell, D., \& Hay, D. C. (1987). Configurational information in face perception. Perception, 16, 747-759.

Yovel, G., \& Kanwisher, N. (2004). Face perception: Domain specific not process specific. Neuron, 44, 889-898.

Yovel, G., \& Kanwisher, N. (2008). The representation of spacing and part-based information are associated for upright faces but dissociated for objects: Evidence from individual differences. Psychonomic Bulletin \& Review, 15, 933-939. doi:10.3758/ PBR.15.5.933

Yovel, G., Paller, K. A., \& Levy, J. (2005). A whole face is more than the sum of its halves: Interactive processing in face perception. Visual Cognition, 12, 337-352. 